\title{
Complementarity Constraints as Nonlinear Equations: Theory and Numerical Experience*
}

\author{
SVEN LEYFFER \\ Mathematics and Computer Science Division \\ Argonne National Laboratory, Argonne, IL 60439, USA. \\ leyffer@mcs . anl .gov \\ September 3, $2003 \quad$ revised February 2, 2005
}

\begin{abstract}
Recently, it has been shown that mathematical programs with complementarity constraints (MPCCs) can be solved efficiently and reliably as nonlinear programs. This paper examines various nonlinear formulations of the complementarity constraints. Several nonlinear complementarity functions are considered for use in MPCC. Unlike standard smoothing techniques, however, the reformulations do not require the control of a smoothing parameter. Thus they have the advantage that the smoothing is exact in the sense that Karush-Kuhn-Tucker points of the reformulation correspond to strongly stationary points of the MPCC. A new exact smoothing of the well-known min function is also introduced and shown to possess desirable theoretical properties. It is shown how the new formulations can be integrated into a sequential quadratic programming solver, and their practical performance is compared on a range of test problems.
\end{abstract}

Keywords: Nonlinear programming, SQP, MPCC, complementarity constraints, NCP functions.

AMS-MSC2000: 90C30, 90C33, 90C55, 49M37, 65K10.

\section{Introduction}

Equilibrium constraints in the form of complementarity conditions often appear as constraints in optimization problems, giving rise to mathematical programs with complementarity constraints (MPCCs). Problems of this type arise in many engineering and economic applications; see the survey [11] and the monographs [24, 26]. The growing collections of test problems $[22,7]$ indicate that this an important area. MPCCs can be

*Preprint ANL/MCS-P1054-0603 
expressed in general as

$$
\begin{array}{cl}
\operatorname{minimize} & f(x) \\
\text { subject to } & c_{\mathcal{E}}(x)=0 \\
& c_{\mathcal{I}}(x) \geq 0 \\
& 0 \leq x_{1} \perp x_{2} \geq 0
\end{array}
$$

where $x=\left(x_{0}, x_{1}, x_{2}\right)$ is a decomposition of the problem variables into controls $x_{0} \in \mathbb{R}^{n}$ and states $x_{1}, x_{2} \in \mathbb{R}^{p}$. The equality constraints $c_{i}(x)=0, i \in \mathcal{E}$ are abbreviated as $c_{\mathcal{E}}(x)=0$, and similarly $c_{\mathcal{I}}(x) \geq 0$ represents the inequality constraints. The notation $\perp$ represents complementarity and means that either a component $x_{1 i}=0$ or the corresponding component $x_{2 i}=0$.

Clearly, more general complementarity constraints can be included in (1.1) by adding slack variables. Adding slacks does not destroy any properties of the MPCC such as constraint qualification or second-order condition. One convenient way of solving (1.1) is to replace the complementarity conditions (1.1d) by

$$
x_{1}, x_{2} \geq 0, \text { and } X_{1} x_{2} \leq 0,
$$

where $X_{1}$ is a diagonal matrix with $x_{1}$ along its diagonal. This transforms the MPCC into an equivalent nonlinear program (NLP) and is appealing because it appears to allow standard large-scale NLP solvers to be used to solve (1.1).

Unfortunately, it has been shown [5, 29] that (1.2) violates the Mangasarian-Fromovitz constraint qualification (MFCQ) at any feasible point. This failure of MFCQ has a number of unpleasant consequences: The multiplier set is unbounded, the central path fails to exist, the active constraint normals are linearly dependent, and linearizations of the NLP can be inconsistent arbitrarily close to a solution. In addition, early numerical experience with (1.2) has been disappointing [2]. As a consequence, solving MPCCs as NLPs has been commonly regarded as numerically unsafe.

Recently, exciting new developments have demonstrated that the gloomy prognosis about the use of (1.2) may have been premature. Standard sequential quadratic programming (SQP) solvers have been used to solve a large class of MPCCs, written as NLPs, reliably and efficiently [16]. This numerical success has motivated a closer investigation of the (local) convergence properties of SQP methods for MPCCs. In [17], it is shown that an SQP method converges locally to strongly stationary points. Anitescu [1] establishes that an SQP method with elastic mode converges locally for MPCCs with (1.2). The key idea is to penalize $X_{1} x_{2} \leq 0$ and consider the resulting NLP, which satisfies MFCQ. Near a strongly stationary point, a sufficiently large penalty parameter can be found, and standard SQP methods converge.

The convergence properties of interior point methods (IPMs) have also received renewed attention. Numerical experiments [3, 28] have shown that IPMs with minor modifications can be applied successfully to solve MPCCs. This practical success has encouraged theoretical studies of the convergence properties of IPMs for MPCCs. Raghunathan and Biegler [27] relax $x_{1}^{T} x_{2} \leq 0$ by a quantity proportional to the barrier parameter, which is driven to zero. Liu and Sun [23] propose a primal-dual IPM that also relaxes the complementarity constraint. 
In this paper, we extend our results of [17] by considering NLP formulations of (1.1) in which the complementarity constraint (1.1d) is replaced by an nonlinear complementarity problem (NCP) function. This gives rise to the following NLP:

$$
\begin{array}{cl}
\operatorname{minimize} & f(x) \\
\text { subject to } & c_{\mathcal{E}}(x)=0 \\
& c_{\mathcal{I}}(x) \geq 0 \\
& x_{1}, x_{2} \geq 0, \Phi\left(x_{1 i}, x_{2 i}\right) \leq 0,
\end{array}
$$

where $\Phi\left(x_{1}, x_{2}\right)$ is the vector of NCP functions, $\Phi\left(x_{1}, x_{2}\right)=\left(\phi\left(x_{11}, x_{21}\right), \ldots, \phi\left(x_{1 p}, x_{2 p}\right)\right)^{T}$, and $\phi$ is any NCP function introduced in the next section. Problem (1.3) is in general nonsmooth because the NCP functions used in (1.3d) are nonsmooth at the origin. We will show that this nonsmoothness does not affect the local convergence properties of the SQP method.

The use of NCP functions for the solution of MPCCs has been considered in $[8,10]$, where a sequence of smoothed NCP reformulation is solved. Our contribution is to show that this smoothing is not required. Thus we avoid the need to control the smoothing parameter that may be problematic in practice. Moreover, the direct use of NCP functions makes our approach exact, in the sense that first-order points of the resulting NLP coincide with strongly stationary points of the MPCC. As a consequence we can prove superlinear convergence under reasonable assumptions.

The paper is organized as follows. The next section reviews the NCP functions that will be used in (1.3d) and their pertinent properties. We also introduce new NCP functions shown to possess certain desirable properties. Section 3 shows the equivalence of first-order points of (1.1) and (1.3). Section 4 formally introduces the SQP algorithm for solving MPCCs. The equivalence of the first-order conditions forms the basis of the convergence proof of the SQP method, presented in Section 5. In Section 6, we examine the practical performance of the different NCP functions on the MacMPEC test set [22]. In Section 7 we summarize our work and briefly discuss open questions.

\section{NCP Functions for MPCCs}

An NCP function is a function $\phi: \mathbb{R}^{2} \rightarrow \mathbb{R}$ such that $\phi(a, b)=0$ if and only if $a, b \geq 0$, and $a b \leq 0$. Several NCP functions can be used in the reformulation (1.3). Here, we review some existing NCP functions and introduce new ones that have certain desirable properties.

1. The bilinear form

$$
\phi_{B L}(a, b)=a b,
$$

which is analytic and has the appealing property that its gradient vanishes at the origin (this makes it consistent with strong stationarity, as will be shown later). It is not an NCP function, however, since $\phi_{B L}(a, b)=0$ does not imply nonnegativity of $a, b$.

2. The Fischer-Burmeister function [12] is given by

$$
\phi_{F B}(a, b)=a+b-\sqrt{a^{2}+b^{2}} .
$$


It is nondifferentiable at the origin, and its Hessian is unbounded at the origin.

3. The min-function [6] is the nonsmooth function

$$
\phi_{\min }(a, b)=\min (a, b) .
$$

It can be written equivalently in terms of the natural residual function [6]:

$$
\phi_{N R}(a, b)=\frac{1}{2}\left(a+b-\sqrt{(a-b)^{2}}\right) .
$$

This function is again nondifferentiable at the origin and along the line $a=b$.

4. The Chen-Chen-Kanzow function [4] is a convex combination of the Fischer-Burmeister function and the bilinear function. For a fixed parameter $\lambda \in(0,1)$, it is defined as

$$
\phi_{C C K}(a, b)=\lambda \phi_{F B}(a, b)+(1-\lambda) a_{+} b_{+},
$$

where $a_{+}=\max (0, a)$. Note that for $a \geq 0, a_{+}=a$; hence, for any method that remains feasible with respect to the simple bounds,

$$
\phi_{C C K}(a, b)=\lambda \phi_{F B}(a, b)+(1-\lambda) \phi_{B L}(a, b)
$$

holds.

We note that all functions (except for (2.4)) are nondifferentiable at the origin. In addition, the Hessian of the Fischer-Burmeister function is unbounded at the origin. This has to be taken into account in the design of robust SQP methods for MPCCs.

The min-function has the appealing property that linearizations of the resulting NLP (1.3) are consistent sufficiently close to a strongly stationary point (see Proposition 3.6). This property motivates the derivation of smooth approximations of the min-function. The first approximation is obtained by smoothing the equivalent natural residual function (2.7) by adding a term to the square root (which causes the discontinuity along $a=b$ ). For a fixed parameter $\sigma_{N R}>1 / 2$, let

$$
\phi_{N R s}(a, b)=\frac{1}{2}\left(a+b-\sqrt{(a-b)^{2}+\frac{a b}{\sigma_{N R}}}\right) .
$$

This smoothing is similar to $[6,10]$, where a positive parameter $4 \mu^{2}>0$ is added to the discriminant. This has the effect that complementarity is satisfied only up to $\mu^{2}$ at the solution. In contrast, adding the term $a b / \sigma_{N R}$, implies that the NCP function remains exact in the sense that $\phi_{N R s}(a, b)=0$ if and only if $a, b \geq 0$ and $a b=0$ for any $\sigma_{N R}>1 / 2$. Figure 2 shows the contours of $\phi_{N R s}(a, b)$ for $\sigma_{N R}=32$ and for the min-function $\left(\sigma_{N R}=\infty\right)$. An interesting observation is that as $\sigma_{N R} \rightarrow \frac{1}{2}$, the smoothed min-function $\phi_{N R s}(a, b)$ becomes the Fischer-Burmeister function (up to a scaling factor).

An alternative way to smooth the natural residual function is to work directly on smoothing the contours of the min-function, which are piecewise constant. The contours can be smoothed by dividing the positive orthant into (for example) three regions as shown in Figure 1. The dashed lines separate the three regions (i) to (iii), and their 


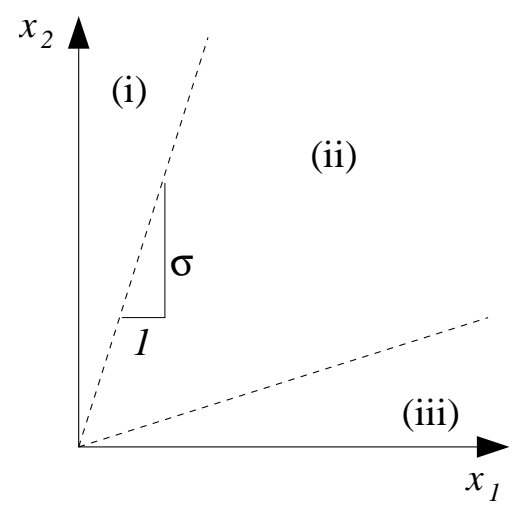

Figure 1: Piecewise regions for smoothing the min-function

slope is $\sigma>1$ and $\sigma^{-1}$, respectively. In regions (i) and (iii), the contours are identical to the min-function. This feature ensures consistency of the linearization. In region (ii), different degrees of smoothing can be applied.

The first smoothed min-function is based on a piecewise linear approximation, given by

$$
\phi_{\text {lin }}(a, b)= \begin{cases}b & b \leq a / \sigma_{l} \\ (a+b) /\left(1+\sigma_{l}\right) & a / \sigma_{l}<b<\sigma_{l} a \\ a & b \geq \sigma_{l} a,\end{cases}
$$

where $\sigma=\sigma_{l}>1$ is the parameter that defines the three regions in Figure 1 . The idea is that close to the axis, the min-function is used, while for values of $a, b$ that are in the center, the decision as to which should be zero is delayed.

The second smoothed min-function is based on the idea of joining the linear parts in sectors (i) and (iii) with circle segments. This gives rise to the following function,

$$
\phi_{\text {qua }}(a, b)= \begin{cases}b & b \leq a / \sigma_{q} \\ \sqrt{\frac{(a-\theta)^{2}+(b-\theta)^{2}}{\left(\sigma_{q}-1\right)^{2}}} & a / \sigma_{q}<b<\sigma_{q} a \\ a & b \geq \sigma_{q} a,\end{cases}
$$

where $\theta$ is the center of the circle, depending on $a, b$, and $\sigma_{q}$ and is given by

$$
\theta=\frac{a+b}{2-\frac{\left(\sigma_{q}-1\right)^{2}}{\sigma_{q}^{2}}}+\sqrt{\left(\frac{a+b}{2-\frac{\left(\sigma_{q}-1\right)^{2}}{\sigma_{q}^{2}}}\right)^{2}-\frac{a^{2}+b^{2}}{2-\frac{\left(\sigma_{q}-1\right)^{2}}{\sigma_{q}^{2}}}}
$$

The contours of both smoothing functions are given in Figure 2. Note that the contours are parallel to the axis in regions (i) and (iii). This fact will be exploited to show that linearizations of the min-function and its two variants remain consistent arbitrarily close to a strongly stationary point. This observation, in effect, establishes a constraint qualification for the equivalent NLP (1.3).

The smoothing also avoids another undesirable property of the min-function: It projects iterates that are far from complementary onto the nearest axis. Close to the axis $a=b$, this projection results in an arbitrary step. Consider, for example, a point $a=99, b=101$. 

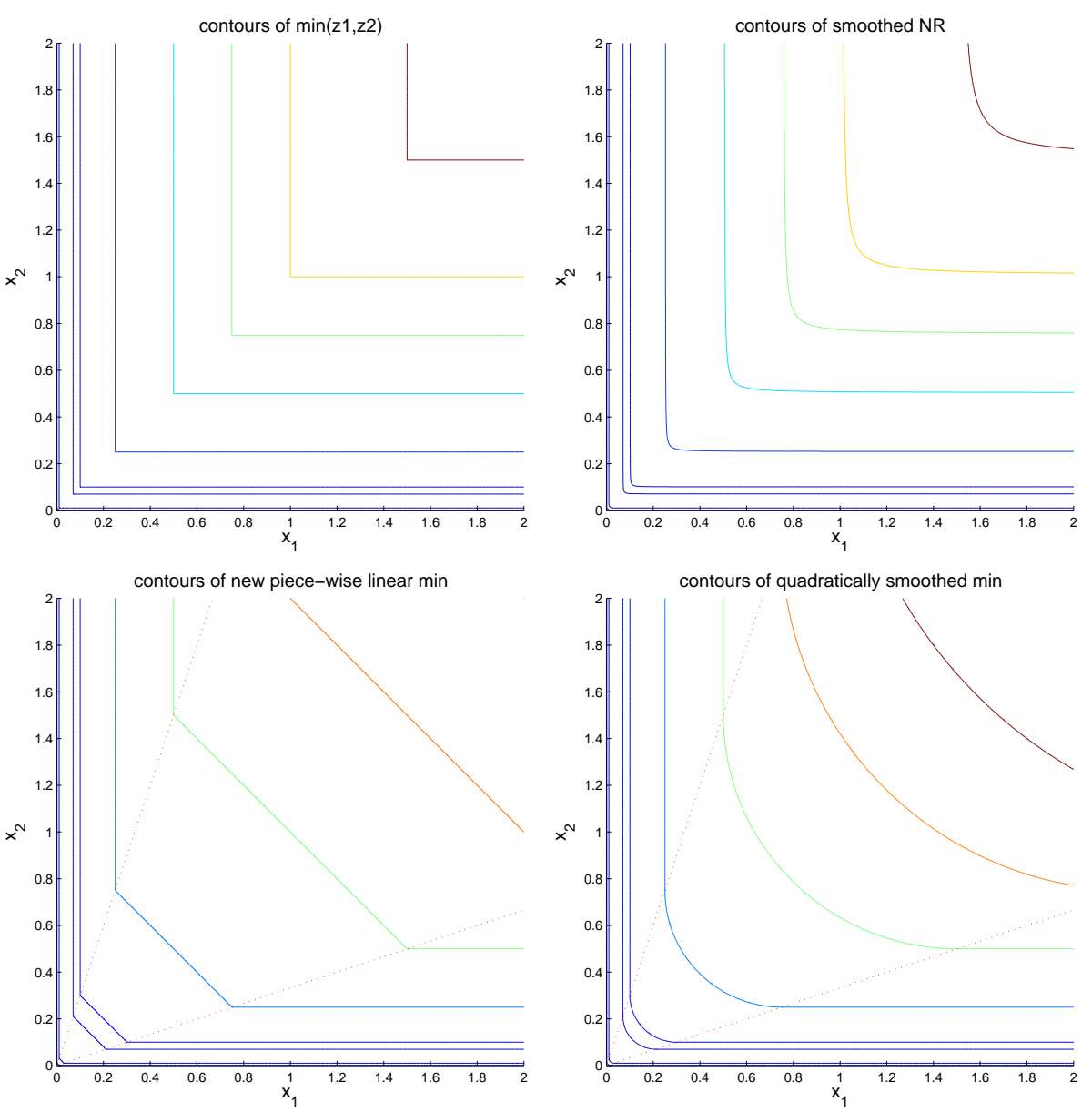

Figure 2: Contours of the min-function, the smoothed natural residual function, the piecewise linear min-function, and the piecewise quadratic with $\sigma_{l}=\sigma_{q}=3$

Linearizing the min-function about this point results in a first-order approximation in which $a=0, b \geq 0$. In contrast, other NCP functions "delay" this decision and can be viewed as smoothing methods.

\section{Equivalence of First-Order Conditions}

This section shows that there exists a one-to-one correspondence between strongly stationary points of the MPCC (1.1) and the first-order stationary points of the equivalent NLP (1.3). We start by reviewing MPCC stationarity concepts. Next, we derive some properties of the linearizations of (1.3d) that play a crucial role in the equivalence of first-order conditions.

\subsection{Strong Stationarity for MPCCs}

The pertinent condition for stationarity for analyzing NLP solvers applied to (1.3) is strong stationarity. The reason is that there exists a relationship between strong stationarity [29] and the Karush-Kuhn-Tucker (KKT) points of (1.3). This relationship has been exploited 
in [17] to establish convergence of SQP methods for MPCCs formulated as NLPs. Strong stationarity is defined as follows.

Definition 3.1 A point $x$ is called strongly stationary if and only if there exist multipliers $\lambda$, $\hat{\nu}_{1}$, and $\hat{\nu}_{2}$ such that

$$
\begin{array}{r}
\nabla f(x)-\nabla c^{T}(x) \lambda-\left(\begin{array}{c}
0 \\
\hat{\nu}_{1} \\
\hat{\nu}_{2}
\end{array}\right)=0 \\
c_{\mathcal{E}}(x)=0 \\
c_{\mathcal{I}}(x) \geq 0 \\
x_{1}, x_{2} \geq 0 \\
x_{1 j}=0 \quad \text { or } x_{2 j}=0 \\
\lambda_{\mathcal{I}} \geq 0 \\
\text { if } x_{1 j}=x_{2 j}=0 \text { then } \hat{\nu}_{1 j} \geq 0 \quad \text { and } \hat{\nu}_{2 j} \geq 0 .
\end{array}
$$

Strong stationarity can be interpreted as the KKT conditions of the relaxed NLP (3.2) at a feasible point $x$. Given two index sets $\mathcal{X}_{1}, \mathcal{X}_{2} \subset\{1, \ldots, p\}$ with

$$
\mathcal{X}_{1} \cup \mathcal{X}_{2}=\{1, \ldots, p\}
$$

denote their respective complements in $\{1, \ldots, p\}$ by $\mathcal{X}_{1}^{\perp}$ and $\mathcal{X}_{2}^{\perp}$. For any such pair of index sets, define the relaxed $N L P$ corresponding to the $M P C C$ (1.1) as

$$
\begin{array}{lllll}
\underset{x}{\operatorname{minimize}} & f(x) & & & \\
\text { subject to } & c_{\mathcal{E}}(x)=0 & & & \\
& c_{\mathcal{I}}(x) \geq 0 & & & \\
& x_{1 j}=0 \quad \forall j \in \mathcal{X}_{2}^{\perp} & \text { and } \quad & x_{1 j} \geq 0 \quad \forall j \in \mathcal{X}_{2} \\
& x_{2 j}=0 \quad \forall j \in \mathcal{X}_{1}^{\perp} \quad \text { and } \quad x_{2 j} \geq 0 \quad \forall j \in \mathcal{X}_{1} .
\end{array}
$$

Concepts such as MPCC constraint qualifications (CQs) and second-order conditions are defined in terms of this relaxed NLP (see, e.g., [17]). Formally, the linear independence constraint qualification (LICQ) is extended to MPCCs as follows:

Definition 3.2 The MPCC (1.1) is said to satisfy an MPCC-LICQ at $x$ if the corresponding relaxed $N L P$ (3.2) satisfies an LICQ.

Next, a second-order sufficient condition (SOSC) for MPCCs is given. Like strong stationarity, it is related to the relaxed NLP (3.2). Let $\mathcal{A}^{*}$ denote the set of active constraints of (3.2) and $\mathcal{A}_{+}^{*} \subset \mathcal{A}^{*}$ the set of active constraints with nonzero multipliers (some could be negative). Let $A$ denote the matrix of active constraint normals, that is,

$$
A=\left[\begin{array}{ccccc} 
& & 0 & & 0 \\
A_{\mathcal{E}}^{*}: & A_{\mathcal{I} \cap \mathcal{A}^{*}}^{*}: & I_{1}^{*} & : & 0 \\
& & 0 & & I_{2}^{*}
\end{array}\right]=:\left[a_{i}^{*}\right]_{i \in \mathcal{A}^{*}},
$$


where $A_{\mathcal{I} \cap \mathcal{A}^{*}}^{*}$ are the active inequality constraint normals and

$$
I_{1}^{*}:=\left[e_{i}\right]_{i \in \mathcal{X}_{1}^{*}} \text { and } I_{2}^{*}:=\left[e_{i}\right]_{i \in \mathcal{X}_{2}^{*}}
$$

are parts of the $p \times p$ identity matrices corresponding to active bounds. Define the set of feasible directions of zero slope of the relaxed NLP (3.2) as

$$
S^{*}=\left\{s \mid s \neq 0, g^{*^{T}} s=0, a_{i}^{*^{T}} s=0, i \in \mathcal{A}_{+}^{*}, a_{i}^{*^{T}} s \geq 0, i \in \mathcal{A}^{*} \backslash \mathcal{A}_{+}^{*}\right\} .
$$

The MPCC-SOSC is defined as follows.

Definition 3.3 A strongly stationary point $z^{*}$ with multipliers $\left(\lambda^{*}, \hat{\nu}_{1}^{*}, \hat{\nu}_{2}^{*}\right)$ satisfies the MPCC-SOSC if for every direction $s \in S^{*}$ it follows that $s^{T} \nabla^{2} \mathcal{L}^{*} s>0$, where $\nabla^{2} \mathcal{L}^{*}$ is the Hessian of the Lagrangian of (3.2) evaluated at $\left(z^{*}, \lambda^{*}, \hat{\nu}_{1}^{*}, \hat{\nu}_{2}^{*}\right)$.

\subsection{Linearizations of the NCP Functions}

All NCP functions with the exception of the bilinear form are nonsmooth at the origin. In addition, the min-function is also nonsmooth along $a=b$, and the linearized min-function is nonsmooth along $a=\sigma^{-1} b$ and $a=\sigma b$. Luckily, SQP methods converge for a simple choice of subgradient.

We start by summarizing some well-known properties of the gradients of the FischerBurmeister function $(2.5)$ for $(a, b) \neq(0,0)$ :

$$
\nabla \phi_{F B}(a, b)=\left(\begin{array}{c}
1-\frac{a}{\sqrt{a^{2}+b^{2}}} \\
1-\frac{b}{\sqrt{a^{2}+b^{2}}}
\end{array}\right) .
$$

It can be shown that $0<1-\frac{a}{\sqrt{a^{2}+b^{2}}}<2$ for all $(a, b) \neq(0,0)$. In addition, if $a>0$ and $b>0$, it can be shown that

$$
\nabla \phi_{F B}(a, 0)=\left(\begin{array}{l}
0 \\
1
\end{array}\right) \text { and } \nabla \phi_{F B}(0, b)=\left(\begin{array}{l}
1 \\
0
\end{array}\right)
$$

Similarly, the gradient of the smoothed natural residual function is

$$
\nabla \phi_{N R s}(a, b)=\frac{1}{2}\left(\begin{array}{c}
1-\frac{a-b+\frac{b}{2 \sigma}}{\sqrt{(a-b)^{2}+\frac{a b}{\sigma}}} \\
1-\frac{b-a+\frac{a}{2 \sigma}}{\sqrt{(a-b)^{2}+\frac{a b}{\sigma}}}
\end{array}\right) .
$$

For $a>0$ and $b>0$, it follows that

$$
\nabla \phi_{N R s}(a, 0)=\left(\begin{array}{c}
0 \\
1-\frac{1}{4 \sigma}
\end{array}\right) \text { and } \nabla \phi_{N R s}(0, b)=\left(\begin{array}{c}
1-\frac{1}{4 \sigma} \\
0
\end{array}\right) \text {. }
$$


Despite the fact that the NCP functions are not differentiable everywhere, it turns out that a particular choice of subgradient gives fast convergence for SQP methods. To show equivalence of the first-order conditions in [17], we exploit the fact that $\nabla \phi_{B L}(0,0)=0$. Fortunately, 0 is a generalized gradient of the other NCP functions, that is, $0 \in \partial \phi(0,0)$. Similarly, we will choose a suitable subgradient for the min-function along $a=b$. With a slight abuse of notation, we summarize the subgradient convention:

Convention 3.4 The following convention is used for subgradients of the nonsmooth NCP functions:

1. $\nabla \phi(0,0)=0$ for any NCP function.

2. $\nabla \phi_{\min }(a, a)=\left(\frac{1}{2}, \frac{1}{2}\right)^{T}$ for the min-function for $a>0$.

3. $\nabla \phi_{\text {lin }}(a, \sigma a)=(0,1)$ and $\nabla \phi_{\text {lin }}\left(a, \sigma^{-1} a\right)=(1,0)$ for the linearized min-function, for $a>0$.

This convention is consistent with the subgradients of the NCP functions and is readily implemented. The most important convention is to ensure that $\nabla \phi(0,0)=0$ because, otherwise, we would not be able to establish equivalence of first-order conditions. The other conventions could be relaxed to allow other subgradients. The convention on the subgradients also has an important practical implication. We have observed convergence to M-stationary, or even C-stationary points that are not strongly stationary for other choices of $0 \neq v \in \partial \phi(0,0)$. Setting $v=0 \in \partial \phi(0,0)$ prevents convergence to such spurious stationary points.

It turns out that a straightforward application of SQP to (1.3) is not very efficient in practice. The reason is that the linearization of the complementarity constraint (1.2) together with the lower bounds has no strict interior. Therefore, we relax the linearization of (1.2). Let $0<\delta, \kappa<1$ be constants, and consider

$$
a \geq 0, b \geq 0, \phi(\hat{a}, \hat{b})+\nabla \phi(\hat{a}, \hat{b})^{T}\left(\begin{array}{c}
a-\hat{a} \\
b-\hat{b}
\end{array}\right) \leq \delta(\min (1, \phi(\hat{a}, \hat{b})))^{1+\kappa} .
$$

Clearly, this is a relaxation of the linearization of (1.2). The following proposition summarizes some useful properties of the linearizations of the NCP functions.

Proposition 3.5 Let $\phi(a, b)$ be one of the functions (2.4)-(2.11). Then it follows that

1. $a, b \geq 0$ and $\phi(a, b) \leq 0$ is equivalent to $0 \leq a \perp b \geq 0$.

2. If $\hat{a}, \hat{b} \geq 0$ and $\hat{a}+\hat{b}>0$, then it follows that the perturbed system of inequalities (3.3) is consistent for any $0 \leq \delta, \kappa \leq 1$. In addition, if $\delta>0$ and $\hat{a}, \hat{b}>0$, then (3.3) has a nonempty interior for the Fischer-Burmeister function, the bilinear function, and the smoothed natural residual function. 
Proof. Part 1 is obvious. For Part 2, consider each NCP function in turn. For the bilinear function $(2.4)$, it readily follows that $(a, b)=(0,0)$ is feasible in (3.3) because for $\hat{a}, \hat{b} \geq 0$, we get

$$
\hat{a} \hat{b}+\nabla \phi_{B L}^{T}\left(\begin{array}{c}
-\hat{a} \\
-\hat{b}
\end{array}\right)=-\hat{a} \hat{b} \leq 0
$$

and clearly, if $\delta>0$, there exists a nonempty interior.

Next consider the Fischer-Burmeister function (2.5), for which (3.3) with $\delta=0$ becomes

$$
\left(1-\frac{\hat{a}}{\sqrt{\hat{a}^{2}+\hat{b}^{2}}}\right) a+\left(1-\frac{\hat{b}}{\sqrt{\hat{a}^{2}+\hat{b}^{2}}}\right) b \leq 0 .
$$

Since the terms in the parentheses are positive, it follows that $(a, b)=0$ is the only point satisfying $a, b \geq 0$ and (3.3). On the other hand, if $\delta>0$, then the right-hand side of (3.3) is positive, and there exists a nonempty interior of $a, b \geq 0$ and (3.3).

For (2.6) and (2.7), it follows for $\hat{a}<\hat{b}$ that (3.3) becomes $a=0, b \geq 0$. The conclusion for $\hat{a}>\hat{b}$ follows similarly. If $\hat{a}=\hat{b}$, then (3.3) becomes $\frac{1}{2} a+\frac{1}{2} b \leq \delta(\min (1, \phi(\hat{a}, \hat{b})))^{1+\kappa}$, and the results follow.

The result for (2.8) follows from the fact that (2.8) is a linear combination of the Fischer-Burmeister function and (2.4).

To show the result for the smoothed min functions, we observe that for $b \leq a / \sigma$ and $b \geq \sigma a$ the functions are identical to the min-function and the result follows. For $a / \sigma<b<\sigma a$, we consider (2.10) and (2.11) in turn. The linearization of (2.10) is equivalent to $a+b \leq 0$, which implies feasibility. It can also be shown that linearization of $(2.11)$ about any point is feasible at the origin $(a, b)=(0,0)$.

The smoothed natural residual function also has feasible linearizations. For (2.9), (3.3) is equivalent to (using $\sigma=\sigma_{N R}$ to simplify the notation)

$$
\phi_{N R s}(\hat{a}, \hat{b})+\left(1-\frac{\hat{a}-\hat{b}+\frac{\hat{b}}{2 \sigma}}{\sqrt{(\hat{a}-\hat{b})^{2}+\frac{\hat{a} \hat{b}}{\sigma}}}\right)(a-\hat{a})+\left(1-\frac{\hat{b}-\hat{a}+\frac{\hat{a}}{2 \sigma}}{\sqrt{(\hat{a}-\hat{b})^{2}+\frac{\hat{a} \hat{b}}{\sigma}}}\right)(b-\hat{b}) \leq 0 .
$$

Rearranging, we have

$$
-\phi_{N R s}(\hat{a}, \hat{b})+\left(1-\frac{\hat{a}-\hat{b}+\frac{\hat{b}}{2 \sigma}}{\sqrt{(\hat{a}-\hat{b})^{2}+\frac{\hat{a} \hat{b}}{\sigma}}}\right) a+\left(1-\frac{\hat{b}-\hat{a}+\frac{\hat{a}}{2 \sigma}}{\sqrt{(\hat{a}-\hat{b})^{2}+\frac{\hat{a} \hat{b}}{\sigma}}}\right) b \leq 0 .
$$

The first term is clearly nonpositive, and it can be shown that the terms multiplying $a$ and $b$ are nonnegative, thus implying consistency and a nonempty interior, even when $\delta=0$.

A disadvantage of the functions (2.4), (2.8), and (2.9) is that arbitrarily close to a strongly stationary point, the linearizations may be inconsistent [17]. The next proposition shows that the min-function and its smoothed versions (2.10) and (2.11) do not have this disadvantage. 
Proposition 3.6 Consider (1.3) using any of the min-functions, (2.6), (2.10), or (2.11), and assume that the MPCC-MFCQ holds at a strongly stationary point. Then it follows that the linearization of (1.3) is consistent for all $x_{1}, x_{2} \geq 0$ sufficiently close to this strongly stationary point.

Proof. Under MPCC-MFCQ, it follows that the linearization of the relaxed NLP (3.2) is consistent in a neighborhood of a strongly stationary point. Now consider the linearization of the min-function near a strongly stationary point, $x^{*}$ say. For components $i$, such that $x_{1 i}^{*}=0<x_{2 i}^{*}$, it follows for any point $x^{k}$ sufficiently close to $x^{*}$ that $0 \leq x_{1 i}^{k}<x_{2 i}^{k}$. Thus, the linearization of the corresponding min-function gives $d_{1 i} \leq-x_{1 i}^{k}$. Together with the lower bound $d_{1 i} \geq-x_{1 i}^{k}$, this is equivalent to $d_{1 i}=-x_{1 i}^{k}$, the linearization of the same component in the relaxed NLP. A similar conclusion holds for components with $x_{1 i}^{*}>0=x_{2 i}^{*}$.

Finally, for components $i$, such that $x_{1 i}^{*}=0=x_{2 i}^{*}$, it follows that the origin $x_{1 i}^{k+1}=$ $x_{2 i}^{k+1}=0$ is feasible (Proposition 3.5). This point is also feasible for the relaxed NLP.

A similar argument can be made for the smoothed min-functions (2.10) and (2.11) by observing that for $x_{1 i}^{*}=0<x_{2 i}^{*}$, there exists a neighborhood where these functions agree with the min-function and for $x_{1 i}^{*}=0=x_{2 i}^{*}$, feasibility follows from Proposition 3.5.

An important consequence of this proposition is that the quadratic convergence proof for MPCCs in [17] can now be applied without the assumption that all QP subproblems are consistent. In this sense, Proposition 3.6 implies that the equivalent NLP (1.3) using the min-functions satisfies a constraint qualification.

\subsection{NCP Functions and Strong Stationarity}

A consequence of the gradient convention is that the gradients of all NCP functions have the same structure. In particular, it follows that for $a, b>0$

$$
\nabla \phi(a, 0)=\left(\begin{array}{c}
0 \\
\tau_{a}
\end{array}\right) \quad \nabla \phi(0, b)=\left(\begin{array}{c}
\tau_{b} \\
0
\end{array}\right) \quad \nabla \phi(a, b)=\left(\begin{array}{c}
\tau_{b} \\
\tau_{a}
\end{array}\right) \quad \text { and } \quad \nabla \phi(0,0)=\left(\begin{array}{c}
0 \\
0
\end{array}\right)
$$

for some parameters $\tau_{a}, \tau_{b}>0$ that depend on $a, b$ and the NCP function. As a consequence, we can generalize the proof of equivalence of first-order conditions from [17] to all NCP functions from Section 2. Let $\Phi\left(x_{1}, x_{2}\right)$ denote the vector of functions $\phi\left(x_{1 i}, x_{2 i}\right)$. The KKT conditions of (1.3) are that there exist multipliers $\mu:=\left(\lambda, \nu_{1}, \nu_{2}, \xi\right)$ such that

$$
\begin{aligned}
& \nabla f(x)-\nabla c(x)^{T} \lambda-\left(\begin{array}{c}
0 \\
\nu_{1} \\
\nu_{2}
\end{array}\right)+\left(\begin{array}{r}
0 \\
\nabla_{x_{1}} \Phi\left(x_{1}, x_{2}\right) \xi \\
\nabla_{x_{2}} \Phi\left(x_{1}, x_{2}\right) \xi
\end{array}\right)=0 \\
& c_{\mathcal{E}}(x)=0 \\
& c_{\mathcal{I}}(x) \geq 0 \\
& x_{1}, x_{2} \geq 0 \\
& \Phi\left(x_{1}, x_{2}\right) \leq 0 \\
& \lambda_{\mathcal{I}} \geq 0 \\
& \nu_{1}, \nu_{2} \geq 0 \\
& \xi \geq 0 \\
& c_{i}(x) \lambda_{i}=x_{1 j} \nu_{1 j}=x_{2 j} \nu_{2 j}=0
\end{aligned} .
$$


There is also a complementarity condition $\xi^{T} \Phi\left(x_{1}, x_{2}\right)=0$, which is implied by feasibility of $x_{1}, x_{2}$ and has been omitted. Note that the choice $\nabla \phi(0,0)=0$ makes (3.4) consistent with strong stationarity, as will be shown next.

Theorem $3.7\left(x^{*}, \lambda^{*}, \hat{\nu}_{1}, \hat{\nu}_{2}\right)$ is a strongly stationary point satisfying (3.1) if and only if there exist multipliers $\left(x^{*}, \lambda^{*}, \nu_{1}^{*}, \nu_{2}^{*}, \xi^{*}\right)$ satisfying the KKT conditions (3.4) of the equivalent NLP (1.3). If $\phi$ is any of the NCP function of Section 2, then

$$
\begin{aligned}
& \hat{\nu}_{1}=\nu_{1}^{*}-\boldsymbol{\tau}_{1} \xi^{*} \\
& \hat{\nu}_{2}=\nu_{2}^{*}-\boldsymbol{\tau}_{2} \xi^{*},
\end{aligned}
$$

where $\boldsymbol{\tau}_{1}$ and $\boldsymbol{\tau}_{2}$ are diagonal matrices of with $\tau_{j}, j=1,2$ along their diagonals. Moreover, $\tau_{j i}=0$, if $x_{1 i}=x_{2 i}=0$ and otherwise satisfies the relationship

$$
\tau_{1 i}= \begin{cases}1 \text { if } x_{2 i}>0 & \text { for }(2.5),(2.6),(2.7),(2.10),(2.11) \\ 1-\frac{1}{4 \sigma} \text { if } x_{2 i}>0 & \text { for }(2.9) \\ x_{2 i} & \text { for }(2.4) \\ \lambda+(1-\lambda) x_{2 i} \text { if } x_{2 i}>0 & \text { for }(2.8)\end{cases}
$$

and

$$
\tau_{2 i}= \begin{cases}1 \text { if } x_{1 i}>0 & \text { for }(2.5),(2.6),(2.7),(2.10),(2.11) \\ 1-\frac{1}{4 \sigma} \text { if } x_{1 i}>0 & \text { for }(2.9) \\ x_{1 i} & \text { for }(2.4) \\ \lambda+(1-\lambda) x_{1 i} \text { if } x_{1 i}>0 & \text { for }(2.8)\end{cases}
$$

Proof. Note that gradients $\nabla \Phi$ have the same structure for all NCP functions used. Then (3.5) follows by comparing (3.4) and (3.1) and taking the gradients of the NCP functions into account.

The failure of MFCQ for (1.3) implies that the multiplier set is unbounded. However, this unboundedness occurs in a special way. The multipliers of (1.3) form a ray, similar to [17], and there exists a multiplier of minimum norm, given by

$$
\begin{array}{lr}
\nu_{1 i}^{*}= & \max \left(\hat{\nu}_{1 i}, 0\right), \\
\nu_{2 i}^{*}= & \max \left(\hat{\nu}_{2 i}, 0\right), \\
\xi_{i}^{*}=-\min \left(\frac{\hat{\nu}_{1 i}}{\tau_{1 i}}, \frac{\hat{\nu}_{2 i}}{\tau_{2 i}}, 0\right) .
\end{array}
$$

This implies the following complementarity conditions for the multipliers

$$
0 \leq \nu_{1 i}^{*} \perp \xi_{i}^{*} \geq 0 \text { and } 0 \leq \nu_{2 i}^{*} \perp \xi_{i}^{*} \geq 0 .
$$

This multiplier will be referred to as the minimal, or basic, multiplier. This term is justified by the observation (to be proved below) that the constraint normals corresponding to nonzero components of the basic multiplier are linearly independent, provided the MPCC satisfies an LICQ. 


\section{An SQP Algorithm for NCP Functions}

This section describes an SQP algorithm for solving (1.3). The algorithm is an iterative procedure that solves a quadratic programming (QP) approximation of (1.3) around the iterate $x^{k}$ for a step $d$ at each iteration:

$$
\left(Q P^{k}\right) \begin{cases}\underset{d}{\operatorname{minimize}} & g^{k^{T}} d+\frac{1}{2} d^{T} W^{k} d \\ \text { subject to } & c_{\mathcal{E}}^{k}+A_{\mathcal{E}}^{k^{T}} d=0 \\ & c_{\mathcal{I}}^{k}+A_{\mathcal{I}}^{k^{T}} d \geq 0 \\ & x_{1}^{k}+d_{1} \geq 0 \\ & x_{2}^{k}+d_{2} \geq 0 \\ & \Phi^{k}+\nabla_{x_{1}} \Phi^{k^{T}} d_{1}+\nabla_{x_{2}} \Phi^{k^{T}} d_{2} \leq \delta\left(\min \left(1, \Phi^{k}\right)\right)^{1+\kappa}\end{cases}
$$

where $\mu^{k}=\left(\lambda^{k}, \nu_{1}^{k}, \nu_{2}^{k}, \xi^{k}\right)$ and $W^{k}=\nabla^{2} \mathcal{L}\left(x^{k}, \mu^{k}\right)$ is the Hessian of the Lagrangian of (1.1):

$$
W^{k}=\nabla^{2} \mathcal{L}\left(x^{k}, \mu^{k}\right)=\nabla^{2} f\left(x^{k}\right)-\sum_{i \in \mathcal{I} \cup \mathcal{E}} \lambda_{i} \nabla^{2} c_{i}\left(x^{k}\right)
$$

Note that the Hessian $W^{k}$ does not include entries corresponding to $\nabla^{2} \Phi$. This omission is deliberate as it avoids numerical difficulties near the origin, where $\nabla^{2} \phi_{F B}$ becomes unbounded. It will be shown that this does not affect the convergence properties of SQP methods.

The last constraint of $\left(Q P^{k}\right)$ is the relaxation of the linearization of the complementarity condition (3.3). We will show that the perturbation does not impede fast local convergence. Formally, the SQP algorithm is defined in Algorithm 1.

Let $k=0, x^{0}$ given

while not optimal do

Solve $\left(Q P^{k}\right)$ for a step $d$

Set $x^{k+1}=x^{k}+d$, and $k=k+1$

Algorithm 1: Local SQP Algorithm for MPCCs

In practice, we also include a globalization scheme to stabilize SQP. In our case, we use a filter [15] and a trust region to ensure convergence to stationary points [18]. The convergence theory of filter methods allows for three possible outcomes [18, Theorem 1]:

(A) The algorithm terminates at a point that is locally infeasible.

(B) The algorithm converges to a Kuhn-Tucker point.

(C) The algorithm converges to a feasible point at which MFCQ fails.

Clearly, (B) cannot happen because (1.3) violates MFCQ at any feasible point. Outcome (A) is typically associated with convergence to a local minimum of the norm of the constraint violation and cannot be avoided unless global optimization techniques are used. Therefore, we deal mainly with outcome (C) if we apply a filter algorithm to MPCC formulated as NLPs (1.3). The next section presents a local convergence analysis of the SQP algorithm applied to (1.3). 


\section{Local Convergence of SQP for MPCCs}

This section establishes superlinear convergence of SQP methods a strongly stationary point under mild conditions. The notation $\boldsymbol{\tau}_{1}, \boldsymbol{\tau}_{2}$ introduced in Theorem 3.7 allows the convergence analysis of all NCP functions to be unified. We note that the presence of the perturbation term $\delta\left(\min \left(1, \Phi^{k}\right)\right)^{1+\kappa}$, with $\kappa<1$, implies that we cannot obtain quadratic convergence in general.

The convergence analysis is concerned with strongly stationary points. Let $x^{*}$ be a strongly stationary point, and denote by $\mathcal{A}\left(x^{*}\right)$ the set of active general constraints:

$$
\mathcal{A}\left(x^{*}\right):=\left\{i \mid c_{i}\left(x^{*}\right)=0\right\}
$$

We also denote the set of active bounds by

$$
\mathcal{X}_{j}\left(x^{*}\right):=\left\{i \mid x_{j i}=0\right\} \text { for } j=1,2
$$

and let $\mathcal{D}\left(x^{*}\right):=\mathcal{X}_{1}\left(x^{*}\right) \cap \mathcal{X}_{2}\left(x^{*}\right)$ be the set of degenerate indices associated with the complementarity constraint.

Assumptions 5.1 We make the following assumptions:

[A0] The subgradients of the NCP functions are computed according to Convention 3.4.

[A1] The functions $f$ and c are twice Lipschitz continuously differentiable.

[A2] (1.1) satisfies an MPCC-LICQ.

[A3] $x^{*}$ is a strongly stationary point that satisfies an MPCC-SOSC.

[A4] $\lambda_{i} \neq 0, \forall i \in \mathcal{E}^{*}, \lambda_{i}^{*}>0, \forall i \in \mathcal{A}^{*} \cap \mathcal{I}$, and either $\nu_{1 j}^{*}>0$ and $\nu_{2 j}^{*}>0, \forall j \in \mathcal{D}^{*}$.

[A5] The QP solver always chooses a linearly independent basis.

We note that [A0] is readily implemented and that assumption [A5] holds for the QP solvers used within snopt [20] and filter [15]. The most restrictive assumptions are [A2] and [A3] because they exclude B-stationary points that are not strongly stationary. This fact is not surprising because it is well known that SQP methods typically converge linearly to such B-stationary points.

It is useful to divide the convergence proof into two parts. First, we consider the case where complementarity holds for some iterate $k$, i.e. $\Phi\left(x_{1}^{k}, x_{2}^{k}\right)=0$. In this case, the SQP method applied to (1.3) is shown to be equivalent to SQP applied to the relaxed NLP (3.2). In the second part, we assume that $\Phi\left(x_{1}^{k}, x_{2}^{k}\right)>0$ for all $k$. Under the additional assumption that all QP approximations remain consistent, superlinear convergence can again be established. 


\subsection{Local Convergence for Exact Complementarity}

In this section we make the additional assumption that

[A6] $\Phi\left(x_{1}^{k}, x_{2}^{k}\right)=0$ and $\left(x^{k}, \mu^{k}\right)$ is sufficiently close to a strongly stationary point.

Assumption [A6] implies that for given index sets $\mathcal{X}_{j}:=\mathcal{X}_{j}\left(x^{k}\right):=\left\{i \mid x_{j i}^{k}=0\right\}, j=$ 1,2 , the following holds:

$$
\begin{array}{lll}
x_{1 j}^{k}=0 & & \forall j \in \mathcal{X}_{2}^{\perp} \\
x_{2 j}^{k}=0 & & \forall j \in \mathcal{X}_{1}^{\perp} \\
x_{1 j}^{k}=0 \quad \text { or } \quad x_{2 j}^{k}=0 & \forall j \in \mathcal{D}=\mathcal{X}_{1} \cap \mathcal{X}_{2} .
\end{array}
$$

In particular, it is not necessary to assume that both $x_{1 i}^{k}=0$ and $x_{2 i}^{k}=0$ for $i \in \mathcal{D}^{*}$. Thus it may be possible that $\mathcal{X}_{1} \neq \mathcal{X}_{1}^{*}$ (and similarly for $\mathcal{X}_{2}$ ). An important consequence of $[\mathbf{A 6}]$ is that $\mathcal{X}_{1}, \mathcal{X}_{2}$ satisfy

$$
\begin{array}{rlrll}
\mathcal{X}_{1}^{*^{\perp}} & \subset & \mathcal{X}_{1}^{\perp} & \subset & \mathcal{X}_{1}^{*^{\perp}} \cup \mathcal{D}^{*} \\
\mathcal{X}_{2}^{*^{\perp}} & \subset & \mathcal{X}_{2}^{\perp} & \subset & \mathcal{X}_{2}^{*^{\perp}} \cup \mathcal{D}^{*} \\
& & \mathcal{D} & \subset & \mathcal{D}^{*}
\end{array}
$$

that is, the indices $\mathcal{X}_{1}^{*^{\perp}}$ and $\mathcal{X}_{2}^{*^{\perp}}$ of the nondegenerate complementarity constraints have been identified correctly.

Next, it is shown that SQP applied to (1.3) is equivalent to SQP applied to the relaxed NLP (3.2). For a given partition $\left(\mathcal{X}_{1}^{\perp}, \mathcal{X}_{2}^{\perp}, \mathcal{D}\right)$, an SQP step for the relaxed NLP (3.2) is obtained by solving the QP

$$
\left(Q P_{R}\left(x^{k}\right)\right)\left\{\begin{array}{llll}
\underset{d}{\operatorname{minimize}} & g^{k^{T}} d+\frac{1}{2} d^{T} W^{k} d & & \\
\text { subject to } & c_{\mathcal{E}}^{k}+A_{\mathcal{E}}^{k^{T}} d=0 & & \\
& c_{\mathcal{I}}^{k}+A_{\mathcal{I}}^{k^{T}} d \geq 0 & & \\
& d_{1 j}=0 & \forall j \in \mathcal{X}_{2}^{\perp} & \text { and } \quad x_{1 j}^{k}+d_{1 j} \geq 0 \quad \forall j \in \mathcal{X}_{2} \\
& d_{2 j}=0 & \forall j \in \mathcal{X}_{1}^{\perp} \quad \text { and } \quad x_{2 j}^{k}+d_{2 j} \geq 0 \quad \forall j \in \mathcal{X}_{1} .
\end{array}\right.
$$

The following proposition shows that SQP applied to the relaxed NLP converges quadratically and identifies the correct index sets $\mathcal{X}_{1}^{*}$ and $\mathcal{X}_{2}^{*}$ in one step. Its proof can be found in [17, Proposition 5.2].

Proposition 5.2 Let [A1]-[A6] hold, and let $x^{k}$ be sufficiently close to $x^{*}$. Consider the relaxed $N L P$ for any index sets $\mathcal{X}_{1}, \mathcal{X}_{2}$ (satisfying (5.1) by virtue of [A6]). Then it follows that

1. there exists a neighborhood $U$ of $\left(z^{*}, \lambda^{*}, \nu_{1}^{*}, \nu_{2}^{*}\right)$ and a sequence of iterates generated by SQP applied to the relaxed NLP (3.2), $\left\{\left(x^{l}, \lambda^{l}, \nu_{1}^{l}, \nu_{2}^{l}\right)\right\}_{l>k}$, that lies in $U$ and converges $Q$-quadratically to $\left(x^{*}, \lambda^{*}, \nu_{1}^{*}, \nu_{2}^{*}\right)$;

2. the sequence $\left\{x^{l}\right\}_{l>k}$ converges $Q$-superlinearly to $x^{*}$; and

3. $\mathcal{X}_{1}^{l}=\mathcal{X}_{1}^{*}$ and $\mathcal{X}_{2}^{l}=\mathcal{X}_{2}^{*}$ for $l>k$. 
Next, it is shown that the QP approximation to the relaxed $\operatorname{NLP}\left(Q P_{R}\left(x^{k}\right)\right)$ and the QP approximation to the NCP formulation $\left(Q P^{k}\right)$ generate the same sequence of steps. The next lemma shows that the solution of $\left(Q P_{R}\left(x^{k}\right)\right)$ is feasible in $\left(Q P^{k}\right)$.

Lemma 5.3 Let Assumptions [A1]-[A6] hold. Then it follows that a step $d$ is feasible in $\left(Q P_{R}\left(x^{k}\right)\right)$ if and only if it is feasible in $\left(Q P^{k}\right)$.

Proof. $\left(Q P_{R}\left(x^{k}\right)\right)$ and $\left(Q P^{k}\right)$ differ only in the way the complementarity constraint is treated. Hence we need only to prove the equivalence of those constraints. Let $j \in \mathcal{X}_{2}^{\perp}$. Then it follows that $x_{1 j}=0$, and $\frac{\partial \Phi^{k}}{\partial x_{1 j}}=\tau_{1 j}>0$, and $\frac{\partial \Phi^{k}}{\partial x_{1 j}}=0$. Hence, $\left(Q P^{k}\right)$ contains the constraints

$$
\tau_{1 j} d_{1 j} \leq 0 \text { and } d_{1 j} \geq 0 \Leftrightarrow d_{1 j}=0
$$

Similarly, we can show that the constraints are equivalent for $j \in \mathcal{X}_{1}^{\perp}$. Let $j \in \mathcal{D}$. Then it follows that $\left(Q P^{k}\right)$ contains the constraints $d_{2 j} \geq 0$ and $d_{1 j} \geq 0$, which are equivalent to the constraints of $\left(Q P_{R}\left(x^{k}\right)\right)$. The equivalence of the feasible sets follows because $\left(\mathcal{X}_{1}^{\perp}, \mathcal{X}_{2}^{\perp}, \mathcal{D}\right)$ is a partition of $\{1, \ldots, p\}$.

The next lemma shows that the solution of the two QPs are identical and that the multipliers are related.

Lemma 5.4 Let Assumptions [A1]-[A6] hold. Let $\left(\lambda, \hat{\nu}_{1}, \hat{\nu}_{2}\right)$ be the Lagrange multipliers of $\left(Q P_{R}\left(x^{k}, \mathcal{X}\right)\right)$ (corresponding to a step $d$ ). Then it follows that the multipliers of $\left(Q P^{k}\right)$, corresponding to the same step $d$ are $\mu=\left(\lambda, \nu_{1}, \nu_{2}, \xi\right)$, where

$$
\begin{aligned}
& \nu_{1 i}= \hat{\nu}_{1 i}>0, \forall i \in \mathcal{D} \\
& \nu_{2 i}= \hat{\nu}_{2 i}>0, \forall i \in \mathcal{D} \\
& \xi_{i}=-\min \left(\frac{\hat{\nu}_{1 i}}{\tau_{1 i}}, \frac{\hat{\nu}_{2 i}}{\tau_{2 i}}, 0\right) \\
& \nu_{1 i}= \hat{\nu}_{1 i}-\xi_{i} \tau_{1 i}, \forall i \in \mathcal{X}_{2}^{\perp} \\
& \nu_{2 i}=\hat{\nu}_{2 i}-\xi_{i} \tau_{2 i}, \quad \forall i \in \mathcal{X}_{1}^{\perp},
\end{aligned}
$$

where $\tau_{j i}$ is given in (3.6-3.7). Conversely, given a solution d and multipliers $\mu$ of $\left(Q P^{k}\right)$, (5.2) shows how to construct multipliers so that $\left(d, \lambda, \widehat{\nu_{1}}, \widehat{\nu_{2}}\right)$ solves $\left(Q P_{R}\left(x^{k}, \mathcal{X}\right)\right)$.

Proof. We equate the first-order conditions of $\left(Q P_{R}\left(x^{k}\right)\right)$ and $\left(Q P^{k}\right)$ and obtain

$$
g^{k}+W^{k} d-A^{k} \lambda=\left(\begin{array}{c}
0 \\
\hat{\nu}_{1} \\
\hat{\nu}_{2}
\end{array}\right)=\left(\begin{array}{c}
0 \\
\nu_{1}-\nabla_{x_{1}} \Phi \xi \\
\nu_{2}-\nabla_{x_{2}} \Phi \xi
\end{array}\right) .
$$

We distinguish three cases:

Case $1(j \in \mathcal{D})$ : It follows from (5.1) that $j \in \mathcal{D}^{*}$, which implies that $\hat{\nu}_{1 j}, \hat{\nu}_{2 j}>0$ for $x^{k}$ sufficiently close to $x^{*}$ by assumption [A4]. Moreover, $\frac{\partial \Phi}{\partial x_{1 j}}=\frac{\partial \Phi}{\partial x_{2 j}}=0$, and hence, $\nu_{1 j}=\hat{\nu}_{1 j}>0, \nu_{2 j}=\hat{\nu}_{2 j}>0$, and $\xi_{j}=0$ are valid multipliers for $\left(Q P^{k}\right)$. 
Case $2\left(j \in \mathcal{X}_{1}^{\perp}\right)$ : We distinguish two further cases. If $j \in \mathcal{D}^{*}$, then a similar argument to Case 1 shows that $\nu_{1 j}=\hat{\nu}_{1 j}>0, \nu_{2 j}=\hat{\nu}_{2 j}>0$, and $\xi_{j}=0$. On the other hand, if $j \in \mathcal{X}_{1}^{*}$, then it follows that $\frac{\partial \Phi}{\partial x_{1 j}}=0$, and $\frac{\partial \Phi}{\partial x_{2 j}}=\tau_{2 j}>0$ is bounded away from zero. Thus, $\nu_{1 j}=\hat{\nu}_{1 j}=0$, and $\nu_{2 j}=\hat{\nu}_{2 j}-\tau_{2 j} \xi_{j}$, and we can always choose $\nu_{2 j}, \xi_{j} \geq 0$. We will show later that the QP solver in fact chooses either $\nu_{2 j}>0$, or $\xi_{j}>0$.

Case $3\left(j \in \mathcal{X}_{2}^{\perp}\right)$ is similar to Case 2 .

Next, it is shown that both QPs have the same solution in a neighborhood of $d=0$; its proof can be found in [17, Lemma 5.6].

Lemma 5.5 The solution $d$ of $\left(Q P_{R}\left(x^{k}\right)\right)$ is the only strict local minimizer in a neighborhood of $d=0$ and its corresponding multipliers $\left(\lambda, \hat{\nu}_{1}, \hat{\nu}_{2}\right)$ are unique. Moreover, $d$ is also the only strict local minimizer in a neighborhood of $d=0$ of $\left(Q P^{k}\right)$.

The next theorem summarizes the results of this section.

Theorem 5.6 If Assumptions [A1]-[A6] hold, then SQP applied to (1.3) generates a sequence $\left\{\left(x^{l}, \lambda^{l}, \nu_{1}^{l}, \nu_{2}^{l}, \xi^{l}\right)\right\}_{l>k}$ that converges Q-quadratically to $\left\{\left(x^{*}, \lambda^{*}, \nu_{1}^{*}, \nu_{2}^{*}, \xi^{*}\right)\right\}$ of (3.4), satisfying strong stationarity. Moreover, the sequence $\left\{x^{l}\right\}_{l>k}$ converges Q-superlinearly to $x^{*}$ and $\Phi\left(x_{1}^{l}, x_{2}^{l}\right)=0$ for all $l \geq k$.

Proof. Under Assumptions [A1]-[A4], SQP converges quadratically when applied to the relaxed NLP (3.2). Lemmas 5.3-5.5 show that the sequence of iterates generated by this SQP method is equivalent to the sequence of steps generated by SQP applied to (1.3). This implies Q-superlinear convergence of $\left\{x^{l}\right\}_{l>k}$. Convergence of the multipliers follows by considering (5.2). Clearly, the multipliers in (5.2a) and (5.2b) converge, as they are just the multipliers of the relaxed NLP, which converge by virtue of Proposition 5.2. Now observe that $(5.2 \mathrm{c})$ becomes

$$
\xi_{i}^{k+1}=-\min \left(\frac{\hat{\nu}_{1 i}^{k+1}}{\tau_{1 i}^{k+1}}, \frac{\hat{\nu}_{2 i}^{k+1}}{\tau_{2 i}^{k+1}}, 0\right) .
$$

The right-hand side of this expression converges because $\hat{\nu}_{1 i}^{k+1}, \hat{\nu}_{2 i}^{k+1}$ converge and the denominators $\tau_{i}^{k+1}$ are bounded away from zero for $i \in \mathcal{X}_{1}^{*^{\perp}}, \mathcal{X}_{2}^{* \perp}$. Finally, (5.2d) and (5.2e) converge by a similar argument.

$\Phi\left(x_{1}^{l}, x_{2}^{l}\right)=0, \forall l \geq k$, follows from the convergence of SQP for the relaxed NLP (3.2) and the fact that SQP retains feasibility with respect to linear constraints. Assumption [A4] ensures that $d_{1 j}^{k}=d_{2 j}^{k}=0, \forall j \in \mathcal{D}^{*}$, since $\nu_{1 j}^{k}, \nu_{2 j}^{k}>0$ for biactive complementarity constraints. Thus SQP will not move out of the corner and stay on the same face.

\subsection{Local Convergence for Nonzero Complementarity}

This section shows that SQP converges superlinearly even if complementarity does not hold at the starting point, that is, if $\Phi\left(x_{1}^{k}, x_{2}^{k}\right)>0$. It is shown in [17] that the QP 
approximation to (1.3) with $x_{1 i} x_{2 i} \leq 0$ can be inconsistent arbitrarily close to a strongly stationary point. Similar examples can be constructed for the NCP functions in Section 2. Only the min-function and its piecewise smooth variations guarantee feasibility of the QP approximation near a strongly stationary point (see Proposition 3.6).

Note that by virtue of the preceding section, any component for which $\phi\left(x_{1 i}^{k}, x_{2 i}^{k}\right)=0$ can be removed from the complementarity constraints and instead be treated as part of the general constraints, as $\phi\left(x_{1 i}^{l}, x_{2 i}^{l}\right)=0$ for all $l \geq k$. Hence, it can be assumed without loss of generality that $\Phi\left(x_{1}^{k}, x_{2}^{k}\right)>0$ for all $k$.

In the remainder of the proof, it is assumed without loss of generality that $\mathcal{X}_{1}^{*^{\perp}}=\emptyset$, that is, the solution can be partitioned as

$$
x_{2}^{*}=\left(\begin{array}{c}
x_{21}^{*} \\
x_{22}^{*}
\end{array}\right)=\left(\begin{array}{c}
0 \\
x_{22}^{*}
\end{array}\right)
$$

where $x_{22}^{*}>0$, and $x_{1}^{*}=0$ is partitioned in the same way. This simplifies the notation in the proof.

SQP methods can take arbitrary steps when encountering infeasible QP approximations. In order to avoid the issue of infeasibility, the following assumption is made that often holds in practice.

[A7] All QP approximations $\left(Q P^{k}\right)$ are consistent for $x^{k}$ sufficiently close to $x^{*}$.

This is clearly an undesirable assumption because it is an assumption on the progress of the method. However, Proposition 3.6 shows that [A7] holds for the NCP reformulations involving the min-function. In addition, it is shown in [17] that [A7] is satisfied for MPCCs with vertical complementarity constraints that satisfy a mixed-P property. Moreover, the use of the perturbation makes it less likely that the SQP method will encounter infeasible QP subproblems.

The key idea behind our convergence result is to show convergence for any "basic" active set. To this end, we introduce the set of active complementarity constraints

$$
\mathcal{C}(x):=\left\{i: \phi\left(x_{1 i}, x_{2 i}\right)=0\right\} .
$$

Let $\mathcal{I}(x):=\mathcal{I} \cap \mathcal{A}(x)$, and let the basic constraints be

$$
\mathcal{B}(x):=\mathcal{E} \cup \mathcal{I}(x) \cup \mathcal{X}_{1}(x) \cup \mathcal{X}_{2}(x) \cup \mathcal{C}(x) .
$$

The set of strictly active constraints (defined in terms of the basic multiplier, $\mu$, see (3.8)) is given by

$$
\mathcal{B}_{+}(x):=\left\{i \in \mathcal{B}(x) \mid \mu_{i} \neq 0\right\} .
$$

Moreover, let $B_{+}^{k}$ denote the matrix of strictly active constraint normals at $x=x^{k}$, namely,

$$
B_{+}^{k}:=\left[a_{i}^{k}\right]_{i \in \mathcal{B}_{+}\left(x^{k}\right)},
$$

where $a_{i}^{k}$ is the constraint normal of constraint $i \in \mathcal{B}_{+}\left(x^{k}\right)$.

The failure of any constraint qualification at a solution $x^{*}$ of the equivalent NLP (1.3) implies that the active constraint normals at $x^{*}$ are linearly dependent. However, the constraint normals corresponding to strictly active constraints are linearly independent, as shown in the following lemma. 
Lemma 5.7 Let Assumptions [A1]-[A4] hold, and let $x^{*}$ be a solution of the MPCC (1.1). Let $\mathcal{I}^{*}$ denote the set of active inequalities $c_{\mathcal{I}}(x)$, and consider the matrix of active constraint normals at $x^{*}$,

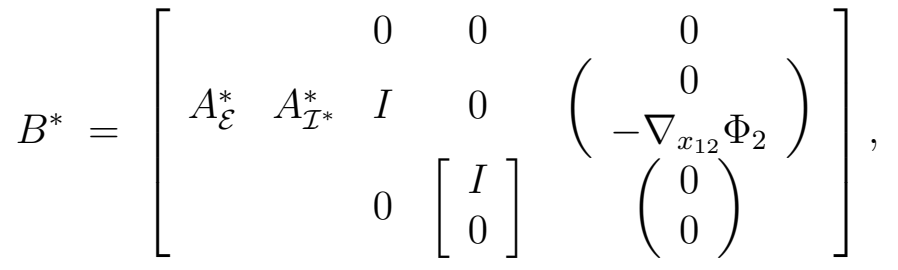

where we have assumed without loss of generality that $\mathcal{X}_{1}^{\perp^{*}}=\emptyset$. The last column is the gradient of the complementarity constraint. Then it follows that $B$ is linearly dependent and that

$$
\operatorname{span}\left\langle\left[\begin{array}{c}
0 \\
I_{2}
\end{array}\right]\right\rangle=\operatorname{span}\left\langle\left[\begin{array}{c}
0 \\
-\nabla_{x_{12}} \Phi_{2}
\end{array}\right]\right\rangle .
$$

Moreover, any submatrix of columns of $B$ has full rank provided that it contains $\left[A_{\mathcal{E}}^{*} A_{\mathcal{I}}^{*}\right]$ and a linearly independent set from the columns in (5.5).

Proof. The structure of the gradient of the NCP functions and (5.3) show that (5.5) holds. Thus $B^{*}$ is linearly dependent. MPCC-LICQ shows that $B^{*}$ without the columns corresponding to the NCP functions has full rank. By choosing a linearly independent subset from the columns in (5.5), we get a basis.

Lemma 5.7 shows that the normals corresponding to the basic multiplier are linearly independent despite the fact that the active normals are linearly dependent. The proof shows that in order to obtain a linearly independent basis, any column of $x_{12}=0$ can be exchanged with the corresponding normal of the complementarity constraint. This matches the observation that the basic multipliers of the simple bounds and the corresponding complementarity constraint are complementary (see (3.9)).

Next, it is shown that for $x^{k}$ sufficiently close to $x^{*}$, if both the normals corresponding to $x_{1 i} \geq 0$ and $\phi\left(x_{1 i}, x_{2 i}\right) \leq 0$ are active, then at the next iteration exact complementarity holds for that component and $\phi\left(x_{1 i}^{l}, x_{2 i}^{l}\right)=0$ and for all subsequent iterations by virtue of Lemma 5.3. Thus, the QP solver cannot continue to choose a basis that is increasingly ill-conditioned.

Lemma 5.8 Let Assumptions [A1]-[A5] hold, and let $x^{k}$ be sufficiently close to $x^{*}$. Partition the NCP function $\Phi=\left(\Phi_{1}, \Phi_{2}\right)^{T}$ in the same way as $x_{1}, x_{2}$ in (5.3). Consider the matrix of active constraint normals at $x^{k}$,

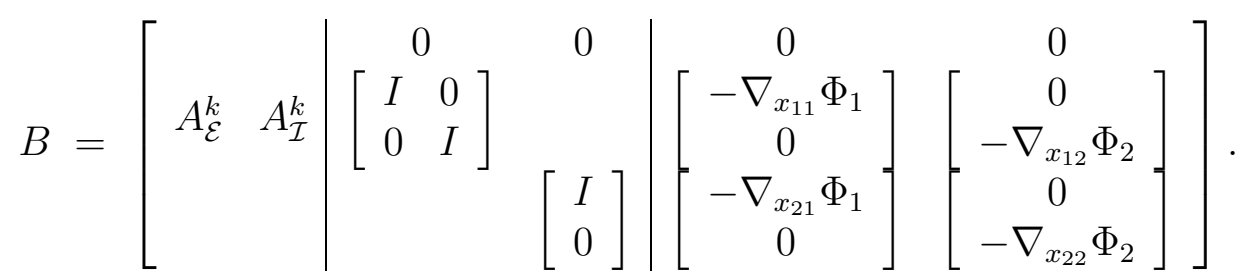

Then it follows that the columns corresponding to the matrix $\nabla_{x} \Phi_{2}$ have the structure $(0,0,-\tau, 0,-\epsilon)^{T}$, where $\tau=\mathcal{O}(1)$ and $\epsilon>0$ is small. If the optimal basis of $\left(Q P^{k}\right)$ 
contains both a column $i$ of $x_{1 i} \geq 0$ and $\phi\left(x_{1 i}, x_{2 i}\right) \leq 0$, then it follows that

$$
x_{1 i}^{k}>0 \text { and } x_{1 i}^{k+1} x_{2 i}^{k+1}=0 .
$$

Moreover, there exists $c>0$ such that

$$
\left\|\left(x^{k+1}, \mu^{k+1}\right)-\left(x^{*}, \mu^{*}\right)\right\| \leq c\left\|\left(x^{k}, \mu^{k}\right)-\left(x^{*}, \mu^{*}\right)\right\| .
$$

Proof. The first part follows by observing that for $x^{k}$ close to $x^{*}, x_{12} \geq 0$ is small and $x_{22}=\mathcal{O}(1)$, which implies the form of the columns. Exchanging them with the corresponding columns of $x_{12} \geq 0$ results in a nonsingular matrix by Lemma 5.7. The second part follows from the nonsingularity assumption [A5] (if $x_{1 i}^{k}=0$, then the basis would be singular) and the fact that if the column corresponding to $x_{1 i} \geq 0$ is basic, then $x_{1 i}^{k+1}=x_{1 i}^{k}+d_{1 i}=0$ holds.

The third part follows by observing that Assumptions [A2] and [A3] imply that the relaxed NLP satisfies an LICQ and a SOSC. Hence, the basis $B$ without the final column gives a feasible point close to $x^{k}$. Denote this solution by $(\hat{x}, \hat{\mu})$, and let the corresponding step be denoted by $\hat{d}$. Clearly, if this step also satisfies the linearization of the complementarity constraint, that is, if

$$
\Phi^{k}+\nabla_{x_{1}} \Phi^{k^{T}} \hat{d}_{1}+\nabla_{x_{2}} \Phi^{k^{T}} \hat{d}_{2} \leq 0
$$

then (5.6) follows by second-order convergence of SQP for the relaxed NLP. If, on the other hand,

$$
\Phi^{k}+\nabla_{x_{1}} \Phi^{k^{T}} \hat{d}_{1}+\nabla_{x_{2}} \Phi^{k^{T}} \hat{d}_{2}>0
$$

then the SQP step of the relaxed NLP is not feasible in $\left(Q P^{k}\right)$. In this case consider the following decomposition of the SQP step. Let

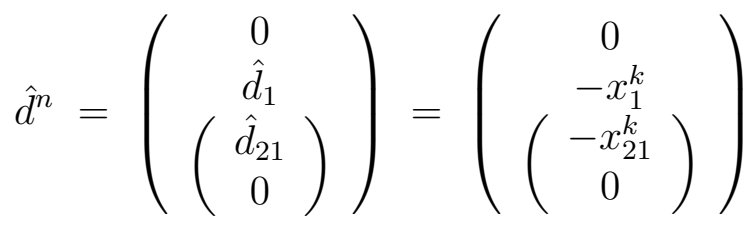

be the normal component, and let $\hat{d}^{t}:=\hat{d}-\hat{d}^{n}$ be the tangential component. Then it follows that the step of $\left(Q P^{k}\right)$ satisfies $d^{k}=\hat{d}^{n}+\sigma \hat{d}^{t}$ for some $\sigma \in[0,1]$, and the desired bound on the distance follows from the convergence of $\hat{d}$.

Thus, if both the normals corresponding to $\phi\left(x_{1 i}, x_{2 i}\right) \leq 0$ and $x_{1 i} \geq 0$ are basic, then $x_{1 i}^{k+1} x_{2 i}^{k+1}=0$ for a point close to $x^{*}$. This component can then be removed from the complementarity constraint, as Lemma 5.3 shows that $x_{1 i}^{k+l} x_{2 i}^{k+l}=0$ for all $l \geq 1$. In the remainder we can therefore concentrate on the case that $x_{1 i}^{k} x_{2 i}^{k}>0$ for all iterates $k$. Next, it is shown that for $x^{k}$ sufficiently close to $x^{*}$, the basis at $x^{k}$ contains the equality constraints $\mathcal{E}$ and the active inequality constraints $\mathcal{I}^{*}$.

Lemma 5.9 Let $x^{k}$ be sufficiently close to $x^{*}$, and let Assumptions [A1]-[A5] and [A7] hold. Then it follows that the optimal basis $B$ of $\left(Q P^{k}\right)$ contains the normals $A_{\mathcal{E}}^{k}$ and $A_{\mathcal{I}^{*}}^{k}$. 
Proof. This follows by considering the gradient of $\left(Q P^{k}\right)$,

$$
0=\nabla f^{k}+W^{k} d^{k}-\nabla c^{k^{T}} \lambda^{k+1}-\left(\begin{array}{c}
0 \\
\nu_{1}^{k+1}-\xi^{k+1} \nabla_{x_{1}} \Phi^{k} \\
\nu_{2}^{k+1}-\xi^{k+1} \nabla_{x_{2}} \Phi^{k}
\end{array}\right)
$$

where $W^{k}$ is the Hessian of the Lagrangian. For $x^{k}$ sufficiently close to $x^{*}$, it follows from [A4] that $\lambda_{i}^{k+1} \neq 0$ for all $i \in \mathcal{E} \cup \mathcal{I}^{*}$.

Thus, as long as the QP approximations remain consistent, the optimal basis of $\left(Q P^{k}\right)$ will be a subset of $B$ satisfying the conditions in Lemma 5.8. The key idea is to show that for any such basis, there exists an equality constraint problem for which SQP converges quadratically. Since there is only a finite number of basis, this implies convergence for SQP.

We now introduce the reduced $N L P$, which is an equality constraint NLP corresponding to a basis with properties as in Lemma 5.8. Assume that $x^{*}$ can be partitioned as in (5.3), and define the reduced NLP as

$$
\begin{array}{ll}
\underset{x}{\operatorname{minimize}} & f(x) \\
\text { subject to } & c_{\mathcal{E}}(x)=0 \\
& c_{\mathcal{I}^{*}}(x)=0 \\
& x_{11}=0 \\
& x_{21}=0 \\
& x_{1 i}=0 \quad \text { or } \quad \Phi\left(x_{1 i}, x_{2 i}\right)=0 \quad \forall i \in \mathcal{X}_{2}^{\perp},
\end{array}
$$

where the last constraint means that either $x_{1 i}=0$ or $\Phi\left(x_{1 i}, x_{2 i}\right)=0$ but not both are present in the reduced NLP. Note that according to $(5.3), \mathcal{X}_{1}^{\perp}=\emptyset$. The key idea will be to relate the reduced NLP to a basis satisfying the conditions of Lemma 5.8. Next, it is shown that any reduced NLP satisfies an LICQ and an SOCS.

Lemma 5.10 Let Assumptions [A1]-[A4] and [A7] hold. Then it follows that any reduced NLP satisfies LICQ and SOSC.

Proof. Lemma 5.8 and the fact that either $x_{1 i}=0$ or $\Phi\left(x_{1 i}, x_{2 i}\right)=0$ are active shows that the normals of the equality constraints of each reduced NLP are linearly independent. The SOSC follows from the MPCC-SOSC and the observation that the MPCC and the reduced NLP have the same null-space.

Thus, applying SQP to the reduced NLP results in second-order convergence. Next, we observe that any nonsingular basis $B$ corresponds to a reduced NLP. Unfortunately, relaxing the complementarity constraints in $\left(Q P^{k}\right)$ means that second-order convergence does not follow directly. However, the particular form of perturbation allows a superlinear convergence result to be established.

Proposition 5.11 Let Assumptions [A1]-[A4] and [A7] hold. Then it follows that an $S Q P$ method that relaxes the complementarity as in $\left(Q P^{k}\right)$ converges superlinearly to $x^{*}$ for any reduced $N L P$. 
Proof. Assume that $\delta=0$, so that no perturbation is used. Lemma 5.10 shows that the reduced NLP satisfy LICQ and SOSC and, therefore, convergence of SQP follows. In particular, it follows that for a given reduced NLP corresponding to a basis $\mathcal{B}$, there exists a constant $c_{\mathcal{B}}>0$ such that

$$
\left\|\left(x^{k+1}, \mu^{k+1}\right)-\left(x^{*}, \mu^{*}\right)\right\| \leq c_{\mathcal{B}}\left\|\left(x^{k}, \mu^{k}\right)-\left(x^{*}, \mu^{*}\right)\right\|^{2} .
$$

If the right-hand side of the complementarity constraint is perturbed (i.e., $\delta>0$ ), then consider the Newton step corresponding to the QP approximation of the relaxed NLP about $x^{k}$. In particular, this step satisfies $d_{N}^{k}=-x_{1}^{k}$, and it follows that the perturbation is $o\left(\left\|d_{N}\right\|\right)$, where $d_{N}$ is the Newton step. Hence, superlinear convergence follows using the Dennis-Moré characterization theorem (e.g., [13, Theorem 6.2.3]).

We note that the SQP method based on $\left(Q P^{k}\right)$ ignores the curvature corresponding to $\phi\left(x_{12}, x_{22}\right)=0$. However, it is easy to extend the proof of Proposition 5.11 to allow $\nabla^{2} \Phi$ to be included. The key idea is to show that the limit of the projected Hessian of $\nabla \Phi^{*}$ is zero. Letting $Z^{k}$ be a basis of the nullspace of $\left(Q P^{k}\right)$, we need to show that $\lim _{k \rightarrow \infty} Z^{k} \nabla^{2} \Phi^{*}=0$, which implies superlinear convergence (see, e.g., [13, Chapter 12.4]). It can be shown that the Hessian of the NCP functions is unbounded in the nullspace of the active constraints of $\left(Q P^{k}\right)$.

Summarizing the results of this section, we obtain the following theorem.

Theorem 5.12 Let Assumptions [A1]-[A5] and [A7] hold. Then it follows that SQP applied to the NLP formulation (1.3) of the MPCC (1.1) converges superlinearly near a solution $\left(x^{*}, \mu^{*}\right)$.

Proof. Proposition 5.11 shows that SQP converges superlinearly for any possible choice of basis $\mathcal{B}$, and Assumption [A7] shows that $\left(Q P^{k}\right)$ is consistent and remains consistent. Therefore, there exists a basis for which superlinear convergence follows. Thus for each basis,

$$
\lim _{k \rightarrow \infty} \frac{\left\|\left(x^{k+1}, \mu^{k+1}\right)-\left(x^{*}, \mu^{*}\right)\right\|}{\left\|\left(x^{k}, \mu^{k}\right)-\left(x^{*}, \mu^{*}\right)\right\|}=0
$$

follows. Since there are a finite number of bases, this condition holds independent of the basis and SQP converges superlinearly.

\subsection{Discussion of Proofs}

Several interesting observations arise from the convergence proofs of the preceding two sections. The curvature of the complementarity constraint $\Phi\left(x_{1}, x_{2}\right)$ can be ignored without losing fast local convergence. This fact is not surprising because the complementarity constraint

$$
0 \leq x_{1} \perp x_{2} \geq 0
$$

has zero curvature at any feasible point with $x_{1 i}+x_{2 i}>0$. At the origin, on the other hand, the curvature is infinite. However, in this case the curvature does not affect convergence, as the reduced Hessian is zero. 
If the min-function (2.6) or its piecewise smooth variants (2.10) or (2.11) are used, then the proof simplifies, as near a strongly stationary point, $\nabla \Phi_{x_{2}}=0$. In addition, the linearizations are consistent even without the perturbation (3.3) and convergence follows from the convergence of the relaxed NLP. This fact can be interpreted as a constraint qualification for the NCP formulations using (2.6) or (2.10) or (2.11) at strongly stationary points.

The conclusions and proofs presented in this section also carry through for linear complementarity constraints but not for general nonlinear complementarity constraints. The reason is that the implication

$$
x_{1 i}^{k} x_{2 i}^{k}=0 \Rightarrow x_{1 i}^{k+1} x_{2 i}^{k+1}=0
$$

holds for linear complementarity problems but not for nonlinear complementarity problems because in general, an SQP method would move off a nonlinear constraint. This is one reason for the introduction of slacks to deal with more general complementarity constraints. In addition, (5.8) can be made to hold in inexact arithmetic by taking care of handling simple bounds appropriately. The same is not true if one expression is a linear equation.

\section{$6 \quad$ Numerical Results}

This section describes our experience with an implementation of the different NCP formulation of the MPCC (1.1) in our sequential quadratic programming solver. Our SQP method promotes global convergence through the use of a filter. The filter accepts a trial point whenever the objective or the constraint violation is improved compared with all previous iterates $[14,15,18]$.

\subsection{Preliminaries}

The solver includes an AMPL [19] interface that introduces slacks to formulate general complementarity constraints in the form (1.1) and handles the reformulation to the NLP (1.3) automatically. The interface also computes the derivatives of the NCP functions and relaxes the linearizations according to (3.3). A user can choose between the various formulations and set parameters such as $\delta, \kappa$ by passing options to the solver.

The test problems come from MacMPEC [22], a collection of some 150 MPCC test problems [16] from a variety of backgrounds and sizes. The numerical tests are performed on a PC with an Intel Pentium 4 processor with $2.5 \mathrm{GHz}$ and $512 \mathrm{~KB}$ RAM running Red Hat Linux version 7.3. The AMPL solver interface is compiled with the Intel $\mathrm{C}++$ compiler version 6.0, and the SQP/MPCC solver is compiled with the Intel Fortran Compiler version 6.0 .

Not all 150 problems in MacMPEC are included in this experiment. We have deliberately left out a number of $32 \times 32$ discretizations of the incidence set identification and packaging problems. These problems are similar to one another (a small number of them are included) but take a long time to run. This is especially true for the formulations that do not lump the complementarity constraint. In this sense, the results would have been even better for the formulation using the scalar product form. 
To determine reasonable values for the various parameters introduced in the definition of the NCP functions, we run a small representative selection of MPCC problems. The overall performance is not very sensitive to a particular parameter choice. No attempt was made to "optimize" the parameter values; rather, we were interested in determining default values that would work well. Table 1 displays the default parameter values.

Table 1: Default parameter values for numerical experiments.

\begin{tabular}{clr} 
Parameter & Description & Default \\
\hline$\delta$ & relaxation of linearization in $(3.3)$ & 0.1 \\
$\kappa$ & relaxation of linearization in $(3.3)$ & 1.0 \\
$\sigma_{N R}$ & smoothing of natural residual $(2.9)$ & 32.0 \\
$\lambda$ & Chen-Chen-Kanzow parameter $(2.8)$ & 0.7 \\
$\sigma_{l}$ & slope of linearized min-function $(2.10)$ & 4.0 \\
$\sigma_{q}$ & slope of quadratic min-function $(2.11)$ & 2.0 \\
\hline
\end{tabular}

While the number of parameters may appear unreasonably large, each formulation requires only three parameters to be set. The choice of $\lambda=0.7$ also agrees with [25], where $\lambda=0.8$ is suggested. Note that since $\delta=0.1$, the Chen-Chen-Kanzow function is relaxed further.

Care has to be taken when computing the smoothed natural residual function (2.9); it can be affected by cancellation error, as the following example illustrates. Suppose $a=10^{4}$ and $b=10^{-4}$ and that single-precision arithmetic is used. Then it follows that

$$
2 \phi_{N R s}(a, b)=\left(10^{4}+10^{-4}\right)-\sqrt{\left(10^{4}-10^{-4}\right)^{2}+\frac{1}{\sigma_{N R}}} \stackrel{\text { float }}{\simeq} 10^{4}-\sqrt{10^{8}}=0,
$$

that is cancellation errors causes (2.9) to declare an infeasible point complementary. This situation can be avoided by employing the same trick used in reformulating the FischerBurmeister function [25], giving rise to

$$
\phi_{N R s}(a, b)=\frac{1}{2} \frac{\left(\frac{4 \sigma_{N R}-1}{\sigma_{N R}}\right)}{a+b+\sqrt{(a-b)^{2}+\frac{a b}{\sigma_{N R}}}} .
$$

Derivative values can be computed in a similarly stable fashion.

\subsection{Performance Plots and Results}

Results are provided in two forms. The performance plots [9] in Figures 3 and 4 show the relative performance of each formulation in terms of iteration count and CPU time. These plots can be interpreted as follows. For every solver $s$ and every problem $p$, the ratio of the number of iterations (or CPU time) of solver $p$ on problem $s$ over the fastest solve for problem $s$ is computed and the base 2 logarithm is taken,

$$
\log _{2}\left(\frac{\# \text { iter }(s, p)}{\operatorname{best\_ iter}(p)}\right) \text {. }
$$



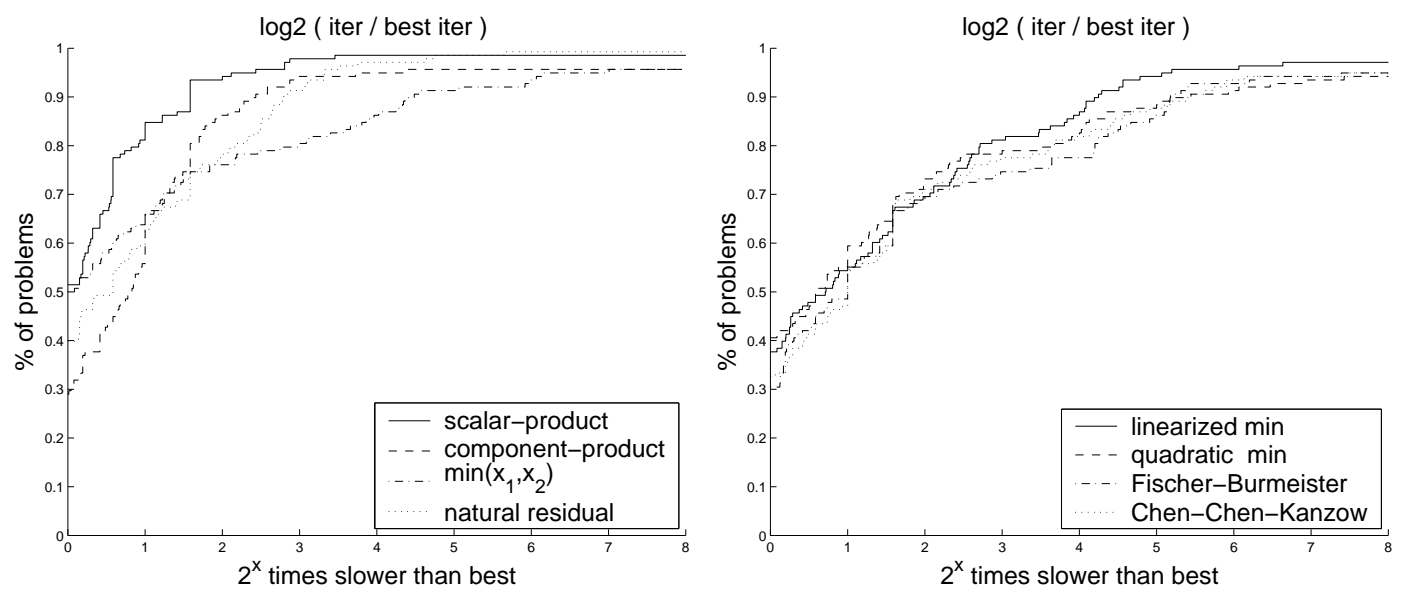

Figure 3: Performance (iterations) plots for different NCP formulations

By sorting these ratios in ascending order for every solver, the resulting plots can be interpreted as the probability that a given solver solves a problem within a certain multiple of the fastest solver.

Failures (see next section) are handled by setting the iteration count and the CPU time to a large number. This strategy ensures that the robustness can also be obtained from the performance plots. The percentage of MPCC problems solved is equivalent to the right asymptote of the performance line for each solver.
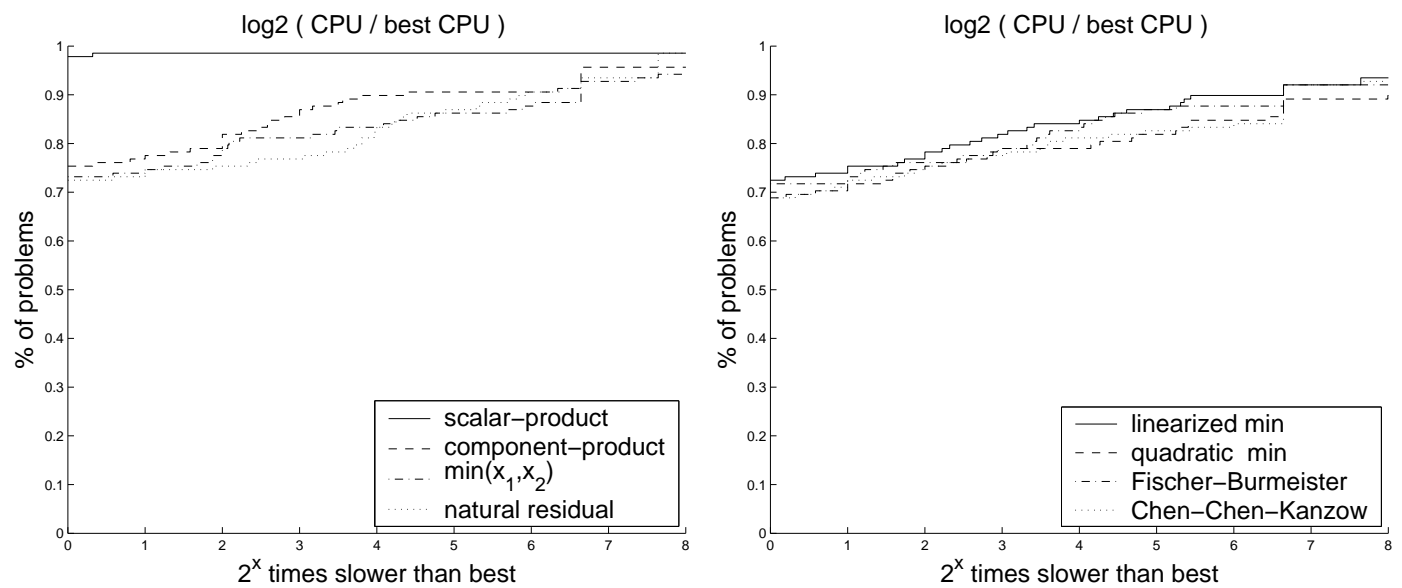

Figure 4: Performance (CPU time) plots for different NCP formulations

\subsection{Failures of the NCP Formulations}

Solving MPCCs as NLPs is surprisingly robust. We observe very few failures, even though many problems are known to violate the assumptions made in this paper. Even the worst NCP formulation failed only on eight problems. Below, we list the problems that failed together with the reason for the failure. 
The NLP solver can fail in three ways. The first failure mode occurs when the trustregion radius becomes smaller than the solver tolerance $(1 \mathrm{E}-6)$ and no further progress can be made. This is referred to in the table below as "TR too small." Such a failure often happens at a solution where the KKT error cannot be reduced to sufficient accuracy. The second failure mode occurs if the QP solver detects inconsistent linearizations near a feasible point. This is referred to as "infeasible QP." Note that the fact that MPCCs violate MFCQ implies that linearizations can become inconsistent arbitrarily close to a feasible point. Third, "iter. limit" refers to failures in which the solver reached its iteration limit (1000) without confirming optimality. The following failures were reported:

1. Scalar product form $x_{1}^{T} x_{2} \leq 0$

2 failures

TR too small : tollmpec1

infeasible QP : design-cent-3

2. Bilinear form $x_{1 i} x_{2 i} \leq 0$

5 failures

infeasible QP : design-cent-3, incid-set1c-32, pack-rig2c-32, pack-rig2p-16

iter. limit : bem-milanc30-s

3. min-function $\min \left(x_{1 i}, x_{2 i}\right) \leq 0$

6 failures

TR too small : ex9.2.2

infeasible QP : pack-comp1p-8, pack-comp1p-16

iter. limit : pack-comp2p-8, pack-comp2p-16, qpec-200-2

4. Linearized min-function (2.10)

4 failures

TR too small : jr2, qpec-200-3

infeasible QP : bem-milanc30-s

iter. limit : qpec-200-2

5. Quadratically smoothed min-function (2.11) 8 failures

TR too small : jr2

infeasible QP : incid-set2c-32

iter. limit : ex9.2.2, gauvin, incid-set1c-32, qpec-100-4, qpec-200-1, qpec-200-3

6. Fischer-Burmeister function (2.5)

7 failures

infeasible QP : design-cent-3, ralphmod

iter. limit : pack-comp1c-8, pack-rig1-16, pack-rig1c-16, pack-rig2-16,

: pack-rig2c-16

7. Smoothed natural residual function (2.9)

1 failures

TR too small : bem-milanc30-s

8. Chen-Chen-Kanzow function (2.8)

5 failures

infeasible QP : pack-comp1p-8, qpec-200-3, pack-comp1c-8, pack-rig2p-16

iter. limit : bem-milanc30-s 
This list contains some problems known not to have strongly stationary limit points. For instance, ex9.2.2, ralph1, and scholtes4 have B-stationary solutions that are not strongly stationary. Problem gauvin has a global minimum at a point where the lower-level problem fails a constraint violation, so the formulation as an MPCC is not appropriate.

In the tests, two problems also gave rise to IEEE errors in the AMPL function evaluations, specifically the Chen-Chen-Kanzow function on pack-rig1-16 and pack-rig1c-32. Since this type of error is caused not by the method but by the model, they are not counted in the errors.

\subsection{Interpretation of the Results}

The results confirm that solving MPCCs as NLPs is very robust. In particular, the scalar product and the smoothed natural residual function are very robust, solving all but two problems and one problem, respectively.

The results for the min-function, on the other hand, are disappointing. Recall that these functions are theoretically attractive because they do not require an additional assumption to be made on the feasibility of QP approximations. This property makes the number of failures $(6 / 4 / 8)$ for the min-function and its smoothed variants disappointing.

The best results in terms of performance and robustness were obtained for the scalar product formulation and the smoothed natural residual function. The performance plots in Figures 3 and 4 clearly show that these formulations are superior. In particular, the scalar product function is significantly faster than any other approach.

The formulation using $x_{1}^{T} x_{2}$ has two main advantages that may explain its superiority. First, it introduces only a single additional constraint, which reduces the size of the NLP to be solved. Moreover, this formulation requires less storage for the QP basis factors. Second, by lumping the complementarity conditions, the formulation allows a certain degree of nonmonotonicity in the complementarity error of each individual $x_{1 i} x_{2 i}$ and reduces the overall complementarity error, $x_{1}^{T} x_{2}$, only.

The worst results in terms of both robustness and efficiency are obtained for the Fischer-Burmeister function and the quadratically smoothed min-function. These formulations fail on seven and eight problems, respectively and are significantly slower than the other formulations. The Chen-Chen-Kanzow function improves on the Fischer-Burmeister function. This observation is not surprising because $\phi_{C C K}$ is a convex combination of the Fischer-Burmeister function and the more successful bilinear formulation. The worse behavior of $\phi_{F B}$ might be due to the fact that its linearized feasible region is smaller than for the bilinear form. This is also supported by the type of failures that can be observed for the Fischer-Burmeister function, which has many infeasible QP terminations.

Analyzing the solution characteristics of the scalar product form, we observe that only four problems have a large value of $\xi$. This fact shows that the SQP method converges to strongly stationary points for the remaining problems, as a bounded complementarity multiplier is equivalent to strong-stationarity (Theorem 3.7). The four problems for which $\xi$ is unbounded are ex9.2.2, ralph1, ralphmod, and scholtes4. The last problem is known to violate an MPCC-MFCQ at its only stationary point, and the limit is B-stationary but not strongly stationary, and SQP converges linearly for this problem [17].

In addition, it can be observed that the complementarity error is exactly zero at most solutions. The reasons for this behavior are as follows: 
1. Complementarity occurs only between variables. Thus, if a lower bound is in the active set, then the corresponding residual can be set to zero even in inexact arithmetic.

2. Many problems in the test set have a solution where $\xi=0$. This indicates that the complementarity constraint $x_{1}^{T} x_{2} \leq 0$ is locally redundant. Hence, exact complementarity is achieved as soon as the SQP method identifies the correct active set.

3. Our QP solver resolves degeneracy by making nearly degenerate constraints exactly degenerate and then employing a recursive procedure to remove degeneracy. This process of making nearly degenerate constraints exactly degenerate forces exact complementarity. Consider any nondegenerate index for which $x_{2 i}^{*}>0=x_{1 i}^{*}$, and assume that $x_{1 i}^{k}>0$ is small. The QP solver resolves the "near" degeneracy between the lower bound $x_{1 i} \geq 0$ and the complementarity constraint by perturbing $x_{1 i}$ to zero. Thus exact complementarity is achieved.

This behavior is reassuring and makes the NLP approach to MPCCs attractive from a numerical standpoint.

\section{Conclusions}

Mathematical programs with complementarity constraints (MPCCs) are an emerging area of nonlinear optimization. Until recently researchers had assumed that the inherent degeneracy of MPCCs makes the application of standard NLP solvers unsafe. In this paper we show how MPCCs can be formulated as NLPs using a range of so-called NCP functions. Two new smoothed min-functions are introduced that exhibit desirable theoretical properties comparable to a constraint qualification.

In contrast to other smoothing approaches, the present formulations are exact in the sense that KKT points of the reformulated NLP correspond to strongly stationary points of the MPCC. Thus there is no need to control a smoothing parameter, which may be problematic.

It is shown that SQP methods exhibit fast local convergence near strongly stationary points under reasonable assumptions. This behavior is observed in practice on a large range of MPCC problems. The numerical results favor a lumped formulation in which all complementarity constraints are lumped into a single constraint. A new smoothed version of the min-function is also shown to be very robust and efficient. On the other hand, results for other standard NCP functions such as the Fischer-Burmeister function are disappointing.

The use of the simple bounds in the reformulation of complementarity (1.2) allows an alternative NLP formulation of the MPCC (1.1). This formulation lumps the nonlinear NCP functions into a single constraint, similar to $x_{1}^{T} x_{2} \leq 0$. Thus, an alternative NLP is 
given by

$$
\begin{array}{ll}
\text { minimize } & f(x) \\
\text { subject to } & c_{\mathcal{E}}(x)=0 \\
& c_{\mathcal{I}}(x) \geq 0 \\
& x_{1}, x_{2} \geq 0 \\
& e^{T} \Phi\left(x_{1}, x_{2}\right) \leq 0 .
\end{array}
$$

It is straightforward to see, that (7.1) is equivalent to (1.1). The convergence proof is readily extended to this formulation. We note that (7.1) has several advantages over (1.3). It reduces the number of constraints in the NLP. Moreover, our experience indicates that the lumped version of the bilinear form, $x_{1}^{T} x_{2} \leq 0$, often performs better than the separate version using $x_{1 i} x_{2 i} \leq 0$. One reason may be that the lumped version allows nonmonotone changes in the complementarity residual in individual variable pairs as long as the overall complementarity is reduced.

Some open questions remain. One question concerns the global convergence of SQP methods from arbitrary starting points. Any approach to this question must take into account the globalization scheme and, in addition, provide powerful feasibility restoration. A related question is whether SQP methods can avoid convergence to spurious stationary points. Such points are sometimes referred to as C-stationary points even though they allow the existence of trivial first-order descent direction. At present, we believe that current SQP methods cannot avoid convergence to C-stationary points. Any attempt to avoid C-stationarity is likely to require algorithmic modifications.

\section{Acknowledgments}

This work was supported by the Mathematical, Information, and Computational Sciences Division subprogram of the Office of Advanced Scientific Computing Research, Office of Science, U.S. Department of Energy, under Contract W-31-109-ENG-38. 


\section{A Detailed Results: Iteration Counts}

\begin{tabular}{|c|c|c|c|c|c|c|c|c|}
\hline Name & $x_{1}^{T} x_{2}$ & $(2.4)$ & $(2.6)$ & $(2.10)$ & $(2.11)$ & $(2.5)$ & $(2.9)$ & $(2.8)$ \\
\hline bard1 & 3 & 4 & 9 & 13 & 2 & 25 & 3 & 8 \\
\hline bard1m & 3 & 4 & 9 & 13 & 2 & 4 & 3 & 7 \\
\hline bard2 & 1 & 1 & 1 & 1 & 1 & 1 & 1 & 1 \\
\hline bard2m & 1 & 1 & 1 & 1 & 1 & 1 & 1 & 1 \\
\hline bard3 & 4 & 4 & 4 & 4 & 4 & 4 & 4 & 4 \\
\hline bard3m & 4 & 4 & 4 & 4 & 4 & 4 & 4 & 4 \\
\hline bar-truss-3 & 10 & 9 & 9 & 9 & 9 & 9 & 9 & 9 \\
\hline bem-milanc30-s & 62 & 1000 & 655 & 111 & 245 & 144 & 410 & 1000 \\
\hline bilevel1 & 2 & 3 & 2 & 3 & 3 & 4 & 3 & 4 \\
\hline bilevel2 & 7 & 2 & 1 & 2 & 5 & 3 & 1 & 2 \\
\hline bilevel3 & 7 & 6 & 6 & 6 & 6 & 6 & 6 & 6 \\
\hline bilin & 2 & 6 & 1 & 3 & 3 & 3 & 5 & 3 \\
\hline dempe & 58 & 58 & 58 & 58 & 58 & 94 & 58 & 58 \\
\hline design-cent-1 & 4 & 4 & 4 & 4 & 4 & 4 & 4 & 4 \\
\hline design-cent-2 & 31 & 21 & 37 & 37 & 29 & 32 & 32 & 60 \\
\hline design-cent-3 & 191 & 164 & 173 & 173 & 173 & 217 & 185 & 163 \\
\hline design-cent-4 & 3 & 4 & 3 & 3 & 3 & 4 & 3 & 4 \\
\hline desilva & 2 & 2 & 2 & 2 & 2 & 2 & 2 & 2 \\
\hline df1 & 2 & 2 & 2 & 2 & 2 & 2 & 2 & 2 \\
\hline ex9.1.1 & 1 & 2 & 1 & 1 & 1 & 3 & 2 & 3 \\
\hline ex9.1.10 & 1 & 1 & 1 & 1 & 1 & 1 & 1 & 1 \\
\hline ex9.1.2 & 2 & 3 & 1 & 3 & 3 & 3 & 3 & 3 \\
\hline ex9.1.3 & 3 & 3 & 1 & 3 & 4 & 3 & 3 & 3 \\
\hline ex9.1.4 & 2 & 2 & 2 & 2 & 2 & 2 & 2 & 2 \\
\hline ex9.1.5 & 3 & 3 & 1 & 3 & 3 & 3 & 3 & 3 \\
\hline ex9.1.6 & 3 & 5 & 2 & 2 & 2 & 4 & 4 & 6 \\
\hline ex9.1.7 & 3 & 3 & 1 & 3 & 3 & 3 & 3 & 3 \\
\hline ex9.1.8 & 1 & 1 & 1 & 1 & 1 & 1 & 1 & 1 \\
\hline ex9.1.9 & 3 & 3 & 2 & 3 & 8 & 3 & 3 & 3 \\
\hline ex9.2.1 & 3 & 4 & 6 & 13 & 6 & 8 & 3 & 8 \\
\hline ex9.2.2 & 22 & 22 & 76 & 71 & 1000 & 238 & 3 & 180 \\
\hline ex9.2.3 & 1 & 1 & 1 & 1 & 1 & 1 & 1 & 1 \\
\hline ex9.2.4 & 3 & 2 & 2 & 2 & 2 & 2 & 2 & 2 \\
\hline ex9.2.5 & 7 & 7 & 1 & 17 & 32 & 35 & 4 & 7 \\
\hline ex9.2.6 & 3 & 2 & 1 & 1 & 2 & 2 & 1 & 2 \\
\hline ex9.2.7 & 3 & 4 & 6 & 13 & 6 & 8 & 3 & 8 \\
\hline ex9.2.8 & 3 & 3 & 1 & 1 & 1 & 4 & 3 & 3 \\
\hline ex9.2.9 & 3 & 3 & 1 & 3 & 3 & 3 & 3 & 3 \\
\hline flp2 & 3 & 3 & 1 & 1 & 1 & 3 & 3 & 1 \\
\hline flp4-1 & 3 & 2 & 2 & 2 & 2 & 2 & 2 & 2 \\
\hline flp4-2 & 3 & 2 & 2 & 2 & 2 & 2 & 2 & 2 \\
\hline flp4-3 & 3 & 2 & 2 & 2 & 2 & 2 & 2 & 2 \\
\hline flp4-4 & 3 & 2 & 2 & 2 & 2 & 2 & 2 & 2 \\
\hline gauvin & 3 & 9 & 71 & 71 & 1000 & 54 & 7 & 6 \\
\hline
\end{tabular}




\begin{tabular}{|c|c|c|c|c|c|c|c|c|}
\hline Name & $x_{1}^{T} x_{2}$ & $(2.4)$ & $(2.6)$ & $(2.10)$ & $(2.11)$ & $(2.5)$ & $(2.9)$ & $(2.8)$ \\
\hline gnash10 & 8 & 8 & 7 & 7 & 7 & 8 & 7 & 8 \\
\hline gnash11 & 8 & 8 & 7 & 7 & 7 & 8 & 7 & 8 \\
\hline gnash12 & 9 & 8 & 8 & 8 & 8 & 8 & 8 & 8 \\
\hline gnash13 & 13 & 9 & 10 & 10 & 9 & 10 & 10 & 11 \\
\hline gnash14 & 10 & 10 & 9 & 9 & 9 & 13 & 10 & 11 \\
\hline gnash15 & 18 & 18 & 41 & 11 & 11 & 9 & 10 & 27 \\
\hline gnash16 & 16 & 14 & 26 & 12 & 10 & 45 & 11 & 14 \\
\hline gnash17 & 17 & 17 & 10 & 10 & 9 & 11 & 10 & 15 \\
\hline gnash18 & 15 & 19 & 55 & 73 & 10 & 184 & 11 & 128 \\
\hline gnash19 & 10 & 19 & 10 & 8 & 8 & 18 & 14 & 25 \\
\hline hakonsen & 10 & 10 & 12 & 12 & 10 & 10 & 10 & 10 \\
\hline hs044-i & 6 & 4 & 2 & 2 & 4 & 4 & 2 & 4 \\
\hline incid-set1-16 & 33 & 139 & 78 & 120 & 493 & 85 & 175 & 66 \\
\hline incid-set1-8 & 34 & 35 & 56 & 56 & 51 & 42 & 73 & 65 \\
\hline incid-set1c-16 & 34 & 89 & 89 & 93 & 168 & 69 & 109 & 86 \\
\hline incid-set1c-32 & 37 & 309 & 102 & 155 & 1000 & 127 & 304 & 161 \\
\hline incid-set1c-8 & 39 & 32 & 43 & 38 & 48 & 35 & 67 & 43 \\
\hline incid-set2-16 & 19 & 37 & 35 & 35 & 35 & 33 & 24 & 33 \\
\hline incid-set2-8 & 48 & 19 & 18 & 18 & 18 & 18 & 18 & 18 \\
\hline incid-set2c-16 & 37 & 36 & 40 & 35 & 305 & 27 & 71 & 32 \\
\hline incid-set2c-32 & 31 & 87 & 71 & 122 & 489 & 71 & 308 & 88 \\
\hline incid-set2c-8 & 24 & 20 & 27 & 23 & 52 & 29 & 25 & 27 \\
\hline jr1 & 1 & 1 & 1 & 1 & 1 & 1 & 1 & 1 \\
\hline jr2 & 7 & 7 & 61 & 66 & 114 & 22 & 3 & 18 \\
\hline kth1 & 1 & 1 & 1 & 1 & 1 & 1 & 1 & 1 \\
\hline kth2 & 2 & 2 & 2 & 2 & 2 & 2 & 2 & 2 \\
\hline kth3 & 4 & 5 & 67 & 67 & 67 & 3 & 2 & 4 \\
\hline liswet1-050 & 1 & 1 & 1 & 1 & 1 & 1 & 1 & 1 \\
\hline liswet1-100 & 1 & 1 & 1 & 1 & 1 & 1 & 1 & 1 \\
\hline liswet1-200 & 1 & 1 & 1 & 1 & 1 & 1 & 1 & 1 \\
\hline nash1 & 3 & 2 & 1 & 1 & 1 & 2 & 1 & 2 \\
\hline outrata31 & 8 & 8 & 7 & 7 & 7 & 8 & 7 & 7 \\
\hline outrata32 & 8 & 9 & 8 & 8 & 8 & 9 & 8 & 8 \\
\hline outrata33 & 7 & 8 & 7 & 7 & 7 & 8 & 7 & 8 \\
\hline outrata34 & 6 & 7 & 6 & 6 & 6 & 7 & 6 & 7 \\
\hline pack-comp1-16 & 20 & 39 & 751 & 64 & 37 & 12 & 68 & 12 \\
\hline pack-comp1-8 & 8 & 30 & 152 & 66 & 24 & 36 & 16 & 36 \\
\hline pack-comp1c-16 & 5 & 38 & 358 & 76 & 40 & 15 & 50 & 15 \\
\hline pack-comp1c-32 & 13 & 2 & 787 & 238 & 217 & 50 & 344 & 50 \\
\hline pack-comp1c-8 & 8 & 19 & 68 & 40 & 14 & 40 & 18 & 41 \\
\hline pack-comp1p-16 & 45 & 72 & 895 & 344 & 81 & 52 & 197 & 31 \\
\hline pack-comp1p-8 & 53 & 64 & 274 & 219 & 87 & 36 & 200 & 172 \\
\hline pack-comp2-16 & 43 & 49 & 442 & 42 & 38 & 44 & 81 & 35 \\
\hline pack-comp2-8 & 8 & 26 & 10 & 18 & 11 & 8 & 10 & 8 \\
\hline pack-comp2c-16 & 15 & 23 & 336 & 76 & 30 & 15 & 17 & 15 \\
\hline pack-comp2c-32 & 7 & 34 & 901 & 193 & 178 & 45 & 175 & 42 \\
\hline pack-comp2c-8 & 6 & 11 & 18 & 15 & 14 & 6 & 13 & 1 \\
\hline
\end{tabular}




\begin{tabular}{|c|c|c|c|c|c|c|c|c|}
\hline Name & $x_{1}^{T} x_{2}$ & $(2.4)$ & $(2.6)$ & $(2.10)$ & $(2.11)$ & $(2.5)$ & $(2.9)$ & $(2.8)$ \\
\hline pack-comp2p-16 & 32 & 64 & 1000 & 190 & 142 & 36 & 232 & 48 \\
\hline pack-comp2p-8 & 60 & 57 & 1000 & 104 & 77 & 34 & 171 & 58 \\
\hline pack-rig1-16 & 64 & 56 & 81 & 120 & 178 & 1000 & 90 & 206 \\
\hline pack-rig1-8 & 7 & 10 & 25 & 13 & 17 & 145 & 13 & 148 \\
\hline pack-rig1c-16 & 11 & 43 & 15 & 57 & 53 & 458 & 19 & 548 \\
\hline pack-rig1c-32 & 18 & 238 & 42 & 302 & 369 & 99 & 181 & 107 \\
\hline pack-rig1c-8 & 6 & 8 & 13 & 13 & 10 & 139 & 9 & 142 \\
\hline pack-rig1p-16 & 28 & 48 & 56 & 164 & 490 & 97 & 118 & 59 \\
\hline pack-rig1p-8 & 14 & 16 & 22 & 25 & 60 & 144 & 29 & 147 \\
\hline pack-rig2-16 & 7 & 11 & 21 & 42 & 119 & 1000 & 30 & 421 \\
\hline pack-rig2-8 & 10 & 16 & 10 & 36 & 38 & 254 & 62 & 253 \\
\hline pack-rig2c-16 & 6 & 11 & 13 & 67 & 96 & 1000 & 34 & 421 \\
\hline pack-rig2c-32 & 11 & 71 & 31 & 187 & 222 & 57 & 551 & 55 \\
\hline pack-rig2c-8 & 6 & 12 & 6 & 15 & 33 & 254 & 23 & 253 \\
\hline pack-rig2p-16 & 10 & 38 & 79 & 367 & 436 & 301 & 86 & 309 \\
\hline pack-rig2p-8 & 20 & 16 & 18 & 46 & 89 & 197 & 20 & 196 \\
\hline portfl1 & 5 & 7 & 4 & 21 & 6 & 76 & 6 & 84 \\
\hline portfl2 & 4 & 6 & 3 & 43 & 8 & 108 & 5 & 162 \\
\hline portfl3 & 4 & 6 & 3 & 8 & 5 & 6 & 10 & 6 \\
\hline portfl4 & 4 & 4 & 5 & 7 & 5 & 50 & 8 & 48 \\
\hline portfl6 & 4 & 6 & 3 & 4 & 5 & 68 & 8 & 66 \\
\hline qpec1 & 3 & 2 & 2 & 2 & 2 & 2 & 2 & 2 \\
\hline qpec-100-1 & 7 & 34 & 114 & 112 & 251 & 253 & 43 & 300 \\
\hline qреc-100-2 & 7 & 24 & 47 & 137 & 427 & 219 & 44 & 43 \\
\hline qреc-100-3 & 6 & 20 & 121 & 137 & 713 & 256 & 27 & 105 \\
\hline qрec-100-4 & 5 & 9 & 103 & 497 & 1000 & 176 & 42 & 78 \\
\hline qрec2 & 2 & 2 & 1 & 1 & 1 & 2 & 1 & 2 \\
\hline qpec-200 & 10 & 24 & 87 & 25 & 1000 & 363 & 38 & 343 \\
\hline qpec-200-2 & 10 & 33 & 1000 & 1000 & 888 & 182 & 114 & 79 \\
\hline qреc-200-3 & 11 & 20 & 160 & 267 & 1000 & 377 & 62 & 357 \\
\hline qреc-200-4 & 5 & 13 & 78 & 95 & 862 & 92 & 34 & 89 \\
\hline ralph1 & 27 & 27 & 70 & 70 & 70 & 368 & 5 & 181 \\
\hline ralph2 & 11 & 21 & 1 & 1 & 1 & 168 & 3 & 179 \\
\hline ralphmod & 7 & 37 & 25 & 46 & 114 & 178 & 48 & 21 \\
\hline scholtes1 & 4 & 3 & 3 & 3 & 3 & 3 & 3 & 3 \\
\hline scholtes2 & 2 & 2 & 2 & 2 & 2 & 2 & 2 & 2 \\
\hline scholtes3 & 4 & 6 & 67 & 67 & 67 & 1 & 1 & 1 \\
\hline scholtes4 & 26 & 28 & 71 & 74 & 74 & 239 & 6 & 181 \\
\hline scholtes5 & 1 & 1 & 1 & 1 & 1 & 1 & 1 & 1 \\
\hline sl1 & 1 & 1 & 1 & 1 & 1 & 1 & 1 & 1 \\
\hline stackelberg1 & 4 & 4 & 4 & 4 & 4 & 4 & 4 & 4 \\
\hline tap-09 & 21 & 23 & 17 & 18 & 18 & 12 & 11 & 23 \\
\hline tap-15 & 28 & 19 & 18 & 12 & 18 & 20 & 19 & 20 \\
\hline tollmpec & 10 & 36 & 22 & 24 & 20 & 79 & 135 & 128 \\
\hline tollmpec1 & 10 & 50 & 20 & 28 & 24 & 379 & 139 & 108 \\
\hline water-FL & 272 & 237 & 235 & 279 & 333 & 256 & 263 & 356 \\
\hline water-net & 131 & 114 & 109 & 125 & 137 & 126 & 190 & 114 \\
\hline
\end{tabular}




\section{B Problem Characteristics}

This appendix lists the problem characteristics obtained with the scalar product formulation. The headings in Appendix B are explained in Table 2. The definition of the degree of degeneracy $d_{1}, d_{2}, d_{m}$ is taken from [21]. The corresponding columns refer to first-level degeneracy, $d_{1}$, second-level degeneracy, $d_{2}$, and mixed-degeneracy, $d_{m}$.

Table 2: Headings for tables in Appendix B.

\begin{tabular}{cl} 
Heading & Description \\
\hline name & problem name in MacMPEC \\
$n$ & number of variables (excluding slacks) \\
$m$ & number of constraints (excluding complementarity) \\
$p$ & number of complementarity constraints \\
$n_{N L P}$ & number of variables after slacks added \\
$k$ & dimension of nullspace at the solution \\
$d_{1}$ & number of indices $i$ with $\lambda_{i}=c_{i}=0$ \\
$d_{2}$ & number of indices $i$ with $x_{1 i}=x_{2 i}=0$ \\
$d_{m}$ & number of indices $i$ with $x_{1 i}=x_{2 i}=0$ and $\left(\nu_{1 i}=0\right.$ or $\left.\nu_{2 i}=0\right)$ \\
compl & complementarity error $\left(x_{1}^{T} x_{2}\right)$ \\
$\xi$ & multiplier of the complementarity constraint $x_{1}^{T} x_{2} \leq 0$ \\
\hline
\end{tabular}




\begin{tabular}{|c|c|c|c|c|c|c|c|c|c|c|}
\hline name & $n$ & $m$ & $p$ & $n_{N L P}$ & $k$ & $d_{1}$ & $d_{2}$ & $d_{m}$ & compl & $\xi$ \\
\hline bard1 & 5 & 4 & 3 & 8 & 0 & 3 & 0 & 0 & 0.00 & 0.762 \\
\hline bard1m & 6 & 4 & 3 & 9 & 0 & 4 & 0 & 0 & 0.00 & 0.762 \\
\hline bard2 & 12 & 9 & 3 & 15 & 0 & 2 & 1 & 0 & 0.00 & 0.00 \\
\hline $\operatorname{bard} 2 \mathrm{~m}$ & 12 & 9 & 3 & 15 & 0 & 2 & 1 & 0 & 0.00 & 0.00 \\
\hline bard3 & 6 & 5 & 1 & 7 & 0 & 2 & 0 & 0 & 0.00 & 0.00 \\
\hline bard3m & 6 & 5 & 3 & 9 & 0 & 2 & 0 & 0 & 0.00 & 1.09 \\
\hline bar-truss-3 & 35 & 34 & 6 & 35 & 0 & 13 & 0 & 0 & 0.00 & 1.45 \\
\hline bem-milanc30-s & 3436 & 3433 & 1464 & 3436 & 1 & 1745 & 1 & 0 & 0.00 & 954. \\
\hline bilevel1 & 10 & 9 & 6 & 12 & 0 & 6 & 0 & 0 & 0.00 & 0.150 \\
\hline bilevel2 & 16 & 13 & 8 & 20 & 1 & 5 & 0 & 0 & $0.294 \mathrm{E}-10$ & 0.00 \\
\hline bilevel3 & 11 & 10 & 3 & 11 & 0 & 5 & 0 & 0 & 0.00 & 1.09 \\
\hline bilin & 8 & 7 & 6 & 14 & 0 & 4 & 0 & 0 & 0.00 & 22.0 \\
\hline dempe & 3 & 2 & 1 & 4 & 0 & 0 & 0 & 0 & 0.00 & $0.571 \mathrm{E}-05$ \\
\hline design-cent-1 & 12 & 11 & 3 & 15 & 0 & 6 & 0 & 0 & 0.00 & 2.17 \\
\hline design-cent-2 & 13 & 15 & 3 & 16 & 0 & 11 & 0 & 0 & 0.00 & 0.00 \\
\hline design-cent-3 & 15 & 11 & 3 & 18 & 0 & 1 & 0 & 1 & 0.00 & $0.313 \mathrm{E}-01$ \\
\hline design-cent- 4 & 22 & 20 & 8 & 30 & 1 & 12 & 0 & 0 & 0.00 & 0.845 \\
\hline desilva & 6 & 4 & 2 & 8 & 0 & 2 & 0 & 2 & 0.00 & 0.00 \\
\hline df1 & 2 & 3 & 1 & 3 & 1 & 1 & 0 & 1 & 0.00 & 0.00 \\
\hline ex9.1.1 & 13 & 12 & 5 & 13 & 0 & 4 & 0 & 0 & 0.00 & 0.00 \\
\hline ex9.1.10 & 11 & 9 & 3 & 11 & 0 & 5 & 0 & 2 & 0.00 & 0.00 \\
\hline ex9.1.2 & 8 & 7 & 2 & 8 & 0 & 4 & 0 & 0 & 0.00 & 0.00 \\
\hline ex9.1.3 & 23 & 21 & 6 & 23 & 0 & 14 & 0 & 1 & 0.00 & 3.20 \\
\hline ex9.1.4 & 8 & 7 & 2 & 8 & 0 & 3 & 0 & 1 & 0.00 & 0.00 \\
\hline ex9.1.5 & 13 & 12 & 5 & 13 & 0 & 8 & 0 & 2 & 0.00 & 10.0 \\
\hline ex9.1.6 & 14 & 13 & 6 & 14 & 0 & 6 & 0 & 1 & 0.00 & 1.56 \\
\hline ex9.1.7 & 17 & 15 & 6 & 17 & 0 & 8 & 0 & 1 & 0.00 & 5.00 \\
\hline ex9.1.8 & 11 & 9 & 3 & 11 & 0 & 5 & 0 & 2 & 0.00 & 0.00 \\
\hline ex9.1.9 & 12 & 11 & 5 & 12 & 0 & 5 & 0 & 1 & 0.00 & 0.444 \\
\hline ex9.2.1 & 10 & 9 & 4 & 10 & 0 & 6 & 0 & 1 & 0.00 & 0.762 \\
\hline ex9.2.2 & 9 & 8 & 3 & 9 & 0 & 3 & 0 & 1 & $0.183 \mathrm{E}-12$ & $0.386 \mathrm{E}+07$ \\
\hline ex9.2.3 & 14 & 13 & 4 & 14 & 0 & 5 & 1 & 0 & 0.00 & 0.00 \\
\hline ex9.2.4 & 8 & 7 & 2 & 8 & 0 & 3 & 0 & 0 & 0.00 & 1.00 \\
\hline ex9.2.5 & 8 & 7 & 3 & 8 & 0 & 3 & 0 & 0 & 0.00 & 6.00 \\
\hline ex9.2.6 & 16 & 12 & 6 & 16 & 2 & 4 & 0 & 2 & $0.168 \mathrm{E}-10$ & 0.500 \\
\hline ex9.2.7 & 10 & 9 & 4 & 10 & 0 & 6 & 0 & 1 & 0.00 & 0.762 \\
\hline ex9.2.8 & 6 & 5 & 2 & 6 & 0 & 3 & 0 & 1 & 0.00 & 0.500 \\
\hline ex9.2.9 & 9 & 8 & 3 & 9 & 0 & 7 & 0 & 0 & $0.100 \mathrm{E}-06$ & 0.00 \\
\hline flp2 & 4 & 2 & 2 & 6 & 1 & 2 & 0 & 1 & 0.00 & 0.987 \\
\hline flp4-1 & 80 & 60 & 30 & 110 & 0 & 30 & 0 & 0 & 0.00 & 0.00 \\
\hline flp4-2 & 110 & 110 & 60 & 170 & 0 & 60 & 0 & 0 & 0.00 & 0.00 \\
\hline flp4-3 & 140 & 170 & 70 & 210 & 0 & 70 & 0 & 0 & 0.00 & 0.00 \\
\hline flp4-4 & 200 & 250 & 100 & 300 & 0 & 100 & 0 & 0 & 0.00 & 0.00 \\
\hline gauvin & 3 & 2 & 2 & 5 & 0 & 1 & 0 & 0 & 0.00 & 0.250 \\
\hline
\end{tabular}




\begin{tabular}{|c|c|c|c|c|c|c|c|c|c|c|}
\hline name & $n$ & $m$ & $p$ & $n_{N L P}$ & $k$ & $d_{1}$ & $d_{2}$ & $d_{m}$ & compl & 3 \\
\hline gnash10 & 13 & 12 & 8 & 13 & 1 & 0 & 0 & 0 & 0.00 & 0.142 \\
\hline gnash11 & 13 & 12 & 8 & 13 & 1 & 0 & 0 & 0 & 0.00 & $0.918 \mathrm{E}-01$ \\
\hline gnash12 & 13 & 12 & 8 & 13 & 1 & 0 & 0 & 0 & 0.00 & $0.397 \mathrm{E}-01$ \\
\hline gnash13 & 13 & 12 & 8 & 13 & 1 & 0 & 0 & 0 & 0.00 & $0.149 \mathrm{E}-01$ \\
\hline gnash14 & 13 & 12 & 8 & 13 & 1 & 0 & 0 & 0 & 0.00 & $0.199 \mathrm{E}-02$ \\
\hline gnash15 & 13 & 12 & 8 & 13 & 0 & 3 & 0 & 0 & 0.00 & 7.65 \\
\hline gnash16 & 13 & 12 & 8 & 13 & 0 & 3 & 0 & 0 & 0.00 & 1.95 \\
\hline gnash17 & 13 & 12 & 8 & 13 & 1 & 4 & 0 & 0 & 0.00 & 1.67 \\
\hline gnash18 & 13 & 12 & 8 & 13 & 1 & 4 & 0 & 0 & 0.00 & 12.7 \\
\hline gnash19 & 13 & 12 & 8 & 13 & 0 & 2 & 0 & 0 & 0.00 & 2.80 \\
\hline hakonsen & 9 & 8 & 4 & 9 & 0 & 2 & 0 & 0 & 0.00 & 0.390 \\
\hline hs044-i & 20 & 14 & 10 & 26 & 0 & 7 & 0 & 1 & 0.00 & 5.69 \\
\hline incid-set1-16 & 485 & 491 & 225 & 485 & 0 & 232 & 0 & 5 & 0.00 & 0.00 \\
\hline incid-set1-8 & 117 & 119 & 49 & 117 & 0 & 54 & 0 & 4 & 0.00 & 0.00 \\
\hline incid-set1c-16 & 485 & 506 & 225 & 485 & 1 & 233 & 1 & 5 & 0.00 & 0.00 \\
\hline incid-set1c-32 & 1989 & 2034 & 961 & 1989 & 4 & 165 & 20 & 0 & 0.00 & 0.00 \\
\hline incid-set1c-8 & 117 & 126 & 49 & 117 & 0 & 59 & 0 & 4 & 0.00 & 0.00 \\
\hline incid-set2-16 & 485 & 491 & 225 & 710 & 3 & 212 & 13 & 0 & 0.00 & 0.00 \\
\hline incid-set2-8 & 117 & 119 & 49 & 166 & 5 & 42 & 7 & 0 & 0.00 & 0.00 \\
\hline incid-set2c-16 & 485 & 506 & 225 & 710 & 0 & 218 & 12 & 0 & 0.00 & 0.00 \\
\hline incid-set2c-32 & 1989 & 2034 & 961 & 2950 & 2 & 937 & 24 & 0 & 0.00 & 0.00 \\
\hline incid-set $2 c-8$ & 117 & 126 & 49 & 166 & 2 & 46 & 6 & 0 & 0.00 & 0.00 \\
\hline jr1 & 2 & 1 & 1 & 3 & 1 & 0 & 0 & 0 & 0.00 & 0.00 \\
\hline jr2 & 2 & 1 & 1 & 3 & 0 & 0 & 0 & 0 & 0.00 & 2.00 \\
\hline kth1 & 2 & 1 & 1 & 2 & 0 & 0 & 1 & 0 & 0.00 & 0.00 \\
\hline kth2 & 2 & 1 & 1 & 2 & 1 & 0 & 0 & 0 & 0.00 & 0.00 \\
\hline kth3 & 2 & 1 & 1 & 2 & 0 & 0 & 0 & 0 & 0.00 & 1.00 \\
\hline liswet1-050 & 152 & 103 & 50 & 202 & 1 & 52 & 0 & 0 & 0.00 & 0.00 \\
\hline liswet1-100 & 302 & 203 & 100 & 402 & 1 & 102 & 0 & 0 & 0.00 & 0.00 \\
\hline liswet1-200 & 602 & 403 & 200 & 802 & 1 & 202 & 0 & 0 & 0.00 & 0.00 \\
\hline nash1 & 6 & 4 & 2 & 8 & 0 & 4 & 0 & 0 & 0.00 & 0.00 \\
\hline outrata31 & 5 & 4 & 4 & 9 & 0 & 0 & 1 & 0 & 0.00 & 0.164 \\
\hline outrata32 & 5 & 4 & 4 & 9 & 1 & 0 & 0 & 0 & 0.00 & 0.168 \\
\hline outrata33 & 5 & 4 & 4 & 9 & 1 & 1 & 0 & 0 & 0.00 & 0.714 \\
\hline outrata34 & 5 & 4 & 4 & 9 & 1 & 1 & 0 & 0 & 0.00 & 2.07 \\
\hline pack-comp1-16 & 467 & 511 & 225 & 692 & 3 & 268 & 0 & 2 & 0.00 & 0.00 \\
\hline pack-comp1-8 & 107 & 121 & 49 & 156 & 0 & 113 & 0 & 0 & 414E-06 & 0.00 \\
\hline pack-comp1c-16 & 467 & 526 & 225 & 692 & 1 & 269 & 0 & 1 & 0.00 & 0.00 \\
\hline pack-comp1c-32 & 1955 & 2138 & 961 & 2916 & 3 & 1108 & 0 & 2 & 0.00 & 0.00 \\
\hline pack-comp1c-8 & 107 & 128 & 49 & 156 & 0 & 120 & 0 & 0 & $0.414 \mathrm{E}-06$ & 0.00 \\
\hline pack-comp1p-16 & 467 & 466 & 225 & 692 & 5 & 223 & 2 & 0 & 0.00 & 0.00 \\
\hline pack-comp1p-8 & 107 & 106 & 49 & 156 & 0 & 83 & 0 & 0 & 0.00 & 0.00 \\
\hline pack-comp2-16 & 467 & 511 & 225 & 692 & 5 & 268 & 0 & 2 & 0.00 & 0.00 \\
\hline pack-comp2-8 & 107 & 121 & 49 & 156 & 5 & 62 & 0 & 2 & 0.00 & 0.00 \\
\hline pack-comp2c-16 & 467 & 526 & 225 & 692 & 4 & 268 & 0 & 2 & 0.00 & 0.00 \\
\hline pack-comp2c-32 & 1955 & 2138 & 961 & 2916 & 16 & 1058 & 0 & 2 & 0.00 & 0.00 \\
\hline pack-comp2c-8 & 107 & 128 & 49 & 156 & 1 & 62 & 0 & 2 & 0.00 & 0.00 \\
\hline
\end{tabular}




\begin{tabular}{|c|c|c|c|c|c|c|c|c|c|c|}
\hline name & $n$ & $m$ & $p$ & $n_{N L P}$ & $k$ & $d_{1}$ & $d_{2}$ & $d_{m}$ & compl & $\xi$ \\
\hline pack-comp2p-16 & 467 & 466 & 225 & 692 & 13 & 223 & 2 & 0 & 0.00 & 0.00 \\
\hline pack-comp2p-8 & 107 & 106 & 49 & 156 & 1 & 47 & 2 & 0 & 0.00 & 0.00 \\
\hline pack-rig1-16 & 380 & 379 & 158 & 485 & 7 & 208 & 0 & 0 & 0.00 & 0.00 \\
\hline pack-rig1-8 & 87 & 86 & 32 & 108 & 6 & 47 & 0 & 0 & 0.00 & 0.00 \\
\hline pack-rig1c-16 & 380 & 394 & 158 & 485 & 4 & 206 & 0 & 0 & 0.00 & 0.00 \\
\hline pack-rig1c-32 & 1622 & 1652 & 708 & 2087 & 2 & 928 & 0 & 0 & $0.763 \mathrm{E}-06$ & 0.00 \\
\hline pack-rig1c-8 & 87 & 93 & 32 & 108 & 5 & 47 & 0 & 0 & 0.00 & 0.00 \\
\hline pack-rig1p-16 & 445 & 444 & 203 & 550 & 3 & 229 & 2 & 0 & 0.00 & 0.00 \\
\hline pack-rig1p-8 & 105 & 104 & 47 & 126 & 5 & 50 & 2 & 0 & 0.00 & 0.00 \\
\hline pack-rig2-16 & 375 & 374 & 149 & 480 & 1 & 203 & 0 & 0 & $0.622 \mathrm{E}-06$ & 0.00 \\
\hline pack-rig2-8 & 85 & 84 & 30 & 106 & 5 & 43 & 0 & 0 & 0.00 & 0.00 \\
\hline pack-rig2c-16 & 375 & 389 & 149 & 480 & 1 & 219 & 0 & 0 & $0.484 \mathrm{E}-06$ & 0.00 \\
\hline pack-rig2c-32 & 1580 & 1610 & 661 & 2045 & 0 & 912 & 0 & 0 & $0.240 \mathrm{E}-06$ & 0.00 \\
\hline pack-rig2c-8 & 85 & 91 & 30 & 106 & 2 & 45 & 0 & 0 & 0.00 & 0.00 \\
\hline pack-rig2p-16 & 436 & 435 & 194 & 541 & 0 & 215 & 1 & 0 & 0.00 & 0.00 \\
\hline pack-rig2p-8 & 103 & 102 & 45 & 124 & 6 & 49 & 2 & 0 & 0.00 & 0.00 \\
\hline portfl1 & 87 & 25 & 12 & 87 & 6 & 6 & 0 & 0 & 0.00 & 0.897 \\
\hline portfl2 & 87 & 25 & 12 & 87 & 0 & 7 & 0 & 0 & 0.00 & 0.682 \\
\hline portfl3 & 87 & 25 & 12 & 87 & 13 & 6 & 0 & 0 & 0.00 & 31.1 \\
\hline portfl4 & 87 & 25 & 12 & 87 & 16 & 5 & 0 & 0 & 0.00 & 114. \\
\hline portfl6 & 87 & 25 & 12 & 87 & 13 & 5 & 0 & 0 & 0.00 & 55.0 \\
\hline qpec1 & 30 & 20 & 20 & 40 & 0 & 10 & 10 & 0 & 0.00 & 0.00 \\
\hline qpec-100-1 & 105 & 102 & 100 & 205 & 0 & 74 & 3 & 0 & 0.00 & 10.1 \\
\hline qpec-100-2 & 110 & 102 & 100 & 210 & 0 & 58 & 4 & 0 & 0.00 & 191. \\
\hline qpec-100-3 & 110 & 104 & 100 & 210 & 0 & 35 & 2 & 0 & 0.00 & 4.45 \\
\hline qpec-100-4 & 120 & 104 & 100 & 220 & 0 & 61 & 4 & 0 & 0.00 & 15.3 \\
\hline qpec2 & 30 & 20 & 20 & 40 & 0 & 0 & 10 & 0 & 0.00 & 0.667 \\
\hline qрec-200-1 & 210 & 204 & 200 & 410 & 0 & 153 & 2 & 0 & 0.00 & 158. \\
\hline qpec-200-2 & 220 & 204 & 200 & 420 & 0 & 118 & 2 & 0 & 0.00 & 3.42 \\
\hline qреc-200-3 & 220 & 208 & 200 & 420 & 0 & 48 & 6 & 0 & 0.00 & 35.5 \\
\hline qрec-200-4 & 240 & 208 & 200 & 440 & 0 & 133 & 7 & 0 & 0.00 & 7.95 \\
\hline ralph1 & 2 & 1 & 1 & 3 & 0 & 0 & 0 & 0 & $0.471 \mathrm{E}-12$ & $0.486 \mathrm{E}+06$ \\
\hline ralph2 & 2 & 1 & 1 & 2 & 1 & 0 & 0 & 0 & $0.313 \mathrm{E}-06$ & 2.00 \\
\hline ralphmod & 104 & 100 & 100 & 204 & 0 & 79 & 2 & 0 & 0.00 & $0.357 \mathrm{E}+08$ \\
\hline scholtes1 & 3 & 1 & 1 & 4 & 0 & 1 & 0 & 0 & 0.00 & 0.00 \\
\hline scholtes2 & 3 & 1 & 1 & 4 & 0 & 0 & 1 & 0 & 0.00 & 0.00 \\
\hline scholtes3 & 2 & 1 & 1 & 2 & 0 & 0 & 0 & 0 & 0.00 & 1.00 \\
\hline scholtes4 & 3 & 3 & 1 & 3 & 0 & 0 & 0 & 0 & $0.161 \mathrm{E}-13$ & $0.525 \mathrm{E}+07$ \\
\hline scholtes5 & 3 & 2 & 2 & 3 & 2 & 0 & 0 & 0 & 0.00 & 0.00 \\
\hline sl1 & 8 & 5 & 3 & 11 & 0 & 5 & 0 & 1 & 0.00 & 0.00 \\
\hline stackelberg1 & 3 & 2 & 1 & 3 & 1 & 0 & 0 & 0 & 0.00 & 0.00 \\
\hline tap-09 & 86 & 68 & 32 & 118 & 8 & 29 & 0 & 2 & 0.00 & $0.687 \mathrm{E}-07$ \\
\hline tap-15 & 194 & 167 & 83 & 277 & 0 & 169 & 0 & 27 & 0.00 & 57.1 \\
\hline tollmpec & 2403 & 2376 & 1748 & 4151 & 1 & 1489 & 1 & 88 & 0.00 & 2.35 \\
\hline tollmpec1 & 2403 & 2376 & 1748 & 4151 & 0 & 2402 & 0 & 86 & 0.00 & 0.00 \\
\hline water-FL & 213 & 160 & 44 & 213 & 3 & 46 & 0 & 0 & 0.00 & $0.163 \mathrm{E}+04$ \\
\hline water-net & 66 & 50 & 14 & 66 & 2 & 15 & 0 & 0 & 0.00 & 0.00 \\
\hline
\end{tabular}




\section{References}

[1] M. Anitescu. On solving mathematical programs with complementarity constraints as nonlinear programs. Preprint ANL/MCS-P864-1200, Mathematics and Computer Science Division, Argonne National Laboratory, Argonne, IL, 2000.

[2] J. F. Bard. Convex two-level optimization. Mathematical Programming, 40(1):15-27, 1988.

[3] H. Benson, D. F. Shanno, and R. V. D. Vanderbei. Interior-point methods for nonconvex nonlinear programming: Complementarity constraints. Technical Report ORFE 02-02, Princeton University, Operations Research and Financial Engineering, 2002.

[4] B. Chen, X. Chen, and C. Kanzow. A penalized Fischer-Burmeister NCP-function. Mathematical Programming, 88(1):211-216, June 2000.

[5] Y. Chen and M. Florian. The nonlinear bilevel programming problem: Formulations, regularity and optimality conditions. Optimization, 32:193-209, 1995.

[6] B. Chen and P. T. Harker. Smooth approximations to nonlinear complementarity problems. SIAM J. Optimization, 7(2):403-420, 1997.

[7] S. P. Dirkse. MPEC world. Webpage, GAMS Development Corp., www.gamsworld.org/mpec/, 2001.

[8] S. P. Dirkse, M. C. Ferris, and A. Meeraus. Mathematical programs with equilibrium constraints: Automatic reformulation and solution via constraint optimization. Technical Report NA-02/11, Oxford University Computing Laboratory, July 2002.

[9] E. D. Dolan and J. Moré. Benchmarking optimization software with cops. Technical Report MCS-TM-246, Mathematics and Computer Science Division, Argonne National Laboratory, Argonne, IL, November 2000.

[10] F. Facchinei, H. Jiang, and L. Qi. A smoothing method for mathematical programs with equilibrium constraints. Mathematical Programming, 85:107-134, 1999.

[11] M. C. Ferris and J. S. Pang. Engineering and economic applications of complementarity problems. SIAM Review, 39(4):669-713, 1997.

[12] A. Fischer. A Newton-type method for positive semi-definite linear complementarity problems. J. Optimization Theory and Applications, 86:585-608, 1995.

[13] R. Fletcher. Practical Methods of Optimization, $2^{\text {nd }}$ edition. John Wiley, Chichester, 1987.

[14] R. Fletcher, N. I. M. Gould, S. Leyffer, Ph. L. Toint, and A. Wächter. Global convergence of trust-region SQP-filter algorithms for general nonlinear programming. SIAM J. Optimization, 13(3):635-659, 2002.

[15] R. Fletcher and S. Leyffer. Nonlinear programming without a penalty function. Mathematical Programming, 91:239-270, 2002. 
[16] R. Fletcher and S. Leyffer. Numerical experience with solving MPECs as NLPs. Numerical Analysis Report NA/210, Department of Mathematics, University of Dundee, Dundee, UK, 2002.

[17] R. Fletcher, S. Leyffer, D. Ralph, and S. Scholtes. Local convergence of SQP methods for mathematical programs with equilibrium constraints. Numerical Analysis Report NA/209, Department of Mathematics, University of Dundee, Dundee, UK, May 2002.

[18] R. Fletcher, S. Leyffer, and Ph. L. Toint. On the global convergence of a filter-SQP algorithm. SIAM J. Optimization, 13(1):44-59, 2002.

[19] R. Fourer, D. M. Gay, and B. W. Kernighan. AMPL: A Modelling Language for Mathematical Programming. Books/Cole-Thomson Learning, 2 edition, 2003.

[20] P. E. Gill, W. Murray, and M. A. Saunders. SNOPT: An SQP algorithm for largescale constrained optimization. SIAM J. Optimization, 12(4):979-1006, 2002.

[21] H. Jiang and D. Ralph. QPECgen, a MATLAB generator for mathematical programs with quadratic objectives and affine variational inequality constraints. Technical Report, University of Melbourne, Department of Mathematics, December 1997.

[22] S. Leyffer. MacMPEC: AMPL collection of MPECs. Webpage, www.mcs.anl.gov/ leyffer/MacMPEC/, 2000.

[23] X. Liu and J. Sun. Generalized stationary points and an interior point method for mathematical programs with equilibrium constraints. Mathematical Programming B, 101(1):231-261, 2004.

[24] Z.-Q. Luo, J.-S. Pang, and D. Ralph. Mathematical Programs with Equilibrium Constraints. Cambridge University Press, Cambridge, UK, 1996.

[25] T. S. Munson, F. Facchinei, M. C. Ferris, A. Fischer, and C. Kanzow. The semismooth algorithm for large scale complementarity problems. INFORMS J. Computing, 13(4):294-311, 2001.

[26] J. Outrata, M. Kocvara, and J. Zowe. Nonsmooth Approach to Optimization Problems with Equilibrium Constraints. Kluwer Academic Publishers, Dordrecht, 1998.

[27] A. Raghunathan and L. T. Biegler. Barrier methods for mathematical programs with complementarity constraints (MPCCs). Technical Report, Carnegie Mellon University, Department of Chemical Engineering, Pittsburgh, PA, December 2002.

[28] A. Raghunathan and L. T. Biegler. MPEC formulations and algorithms in process engineering. Technical Report, Carnegie Mellon University, Department of Chemical Engineering, Pittsburgh, PA, 2002.

[29] H. Scheel and S. Scholtes. Mathematical program with complementarity constraints: Stationarity, optimality and sensitivity. Mathematics of Operations Research, 25:122, 2000 . 
The submitted manuscript has been created by the University of Chicago as Operator of Argonne National Laboratory ("Argonne") under Contract No. W-31-109-ENG-38 with the U.S. Department of Energy. The U.S. Government retains for itself, and others acting on its behalf, a paid-up, nonexclusive, irrevocable worldwide license in said article to reproduce, prepare derivative works, distribute copies to the public, and perform publicly and display publicly, by or on behalf of the Government. 